\title{
I DIVERSI APPROCCI METODOLOGICI NELL'OPERA FISICA DI ARISTOTELE
}

Lucia Palpacelli*

\begin{abstract}
In questo intervento mi propongo di dare un quadro del metodo aristotelico nelle opere fisiche e biologiche, rilevando la duttilità metodologica tipica di Aristotele e la varietà di schemi interpretativi della realtà che tale metodologia sottintende, nel contesto di una scienza come la fisica che offre un vastissimo campo di applicazione ed è definita dallo Stagirita la scienza di «ciò che accade sempre o

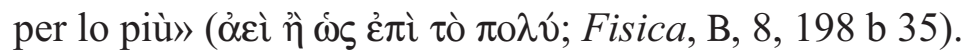

L'argomentazione si articola come segue:

1. la definizione del concetto aristotelico di physis e di scienza della natura;

2. la delineazione del campo di indagine del physikos, definito come colui che si occupa della forma unita alla materia;

3. l'individuazione, nel pensiero aristotelico, di un approccio gnoseologico di fondo che consiste nel moltiplicare gli schemi esplicativi della realtà, per poterla comprendere e spiegare in tutta la sua complessità;

4. la valutazione della ricaduta di tale ricchezza gnoseologica a livello metodologico: è, infatti, possibile spiegare la radicale duttilità metodologica aristotelica come un'espressione, sul piano del metodo, di questo suo atteggiamento gnoseologico.

5. la caratterizzazione del metodo (o dei metodi) da applicare alla scienza della natura: si individua, infatti, nell'opera

* Doutora em Storia della Filosofia pela Università degli Studi di Macerata, Italia. E-mail: lucia.palpacelli@virgilio.it
\end{abstract}


fisica la compresenza e la collaborazione di due movenze costanti che rispondono a finalità diverse:

5.1. l'analisi degli endoxa dei predecessori, terreno sul quale Aristotele opera dialetticamente;

5.2. il procedimento che va dal $\kappa \alpha \theta \hat{\lambda} \lambda$ ov al $\kappa \alpha \theta$ 'ย̌ $\kappa \alpha \sigma \tau \alpha$ nelle sue varie articolazioni che delineano, in contesti diversi, tanto un metodo induttivo quanto un metodo deduttivo.

PAROLE CHIAVI: Metodo, physis. Endoxa e empeiria. Molteplicità dei punti di vista

\begin{abstract}
In this article we present an outline of Aristotle's method in his physical and biological works, pointing out the ductile method, typical of Aristotle, and the variety of interpretation schemes of the reality, implied by this method. This an important approach in the context of a science, like physics, which offers a very wide range of applications and is defined by Aristotle as the science of «what happens always or

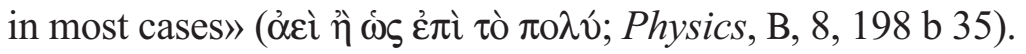

The argumentation is organized as follows:

1. the definition of Aristotle's concept of physis and natural science;

2. the outline of physikos' research field, defined as who looks after the form joined to the matter;

3. the gnoseological procedure of multiplying the explanatory schemes of reality, in order to understand it in all of its complexity;

4. the interpretation of this gnoseological approach as the underlying explanation of the methodological variety which characterize Aristotle's work;
\end{abstract}


5. the characterization of the method (or methods) to be applied to the natural science: in fact, in Aristotle's physical work it's possible to recognize both the presence and the collaboration of two constant movements, that respond to different goals:

5.1. the analysis of the predecessors' endoxa, field where Aristotle works in a dialectic way;

5.2. the procedure that goes from $\kappa \alpha \theta$ ó $\lambda$ ov to

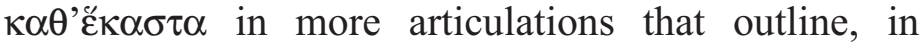
different contexts, as an inductive method as a deductive method.

KEYWORDS: Method. Physis. Endoxa and empeiria. Multiplicity of the points of view

\section{Il concetto aristotelico della physis e la scienza della natura}

L'interesse aristotelico per la physis è certamente uno dei tratti che meglio caratterizzano la sua ricerca filosofica, testimoniato dal fatto che "gli scritti fisici costituiscono circa la metà dei trattati sistematici ancora esistenti". ${ }^{1}$ Del resto,

[...] la distinzione di una problematica fisica, dopo le acquisizioni platoniche, si imponeva strutturalmente: se due sono i piani della realtà, $\mathrm{o}$, per esprimerci in termini più aristotelici, se esistono due diversi generi di sostanze strutturalmente distinti, il genere soprasensibile e il genere sensibile, allora necessariamente diverse dovranno essere altresì le scienze aventi queste due diverse realtà

\footnotetext{
${ }^{1} \mathrm{~A}$. Mansion, Introduction à la physique aristotélicienne, Éditions de l'Institut Supérieur de Philosophie, Louvain - La - Neuve, 1913, $1945,{ }^{2}$ p. 44; egli nota, inoltre, che "delle circa 1450 pagine che comprendono i due volumi del testo greco, la serie ne copre 436", anche se si eliminano i cosiddetti trattati biologici e le opere di dubbia autenticità.
}

Educ. e Filos. Uberlândia, v. 23, n 45, p. 101-164, jan./jun. 2009. 
come oggetto di indagine. ${ }^{2}$

Ora, tale affermazione segna un fortissimo scarto rispetto alla concezione della fisica presocratica, nella quale la physis coincideva con l'intera realtà. Questo, nell'orizzonte aristotelico (cioè postplatonico) non può più essere ammesso e la physis aristotelica viene, infatti, a coincidere con l'essere sensibile e, dunque, "natura vorrà dire prevalentemente natura sensibile". ${ }^{3}$

A rendere testimonianza di questa evoluzione semantica e filosofica del termine vúøı è, del resto, lo stesso Aristotele che, in Metafisica, $\Delta, 4$, offre un vasto spettro dei significati di tale termine:

physis si dice $<1>$ in un senso, la generazione delle cose vengono generate (così, se si pronuncia come lunga la lettera " $y$ " del termine

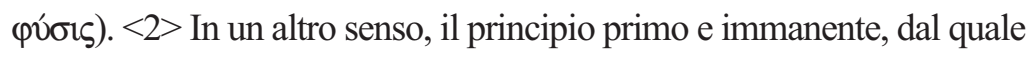
dipende la generazione di ciò che è generato. $<3>$ Inoltre, il principio

${ }^{2}$ G. Reale, Introduzione a Aristotele, Editori Laterza, Roma-Bari, 1974, 19912, p. 73; sulla stessa linea anche Migliori precisa che "il fondamentale guadagno platonico, la divisione tra mondo sensibile e mondo soprasensibile, consente di uscire da quella 'metafisica implicita' che segna l'elaborazione dei 'fisici' precedenti” (M. Migliori, Rapporti tra la Metafisica e il De generatione et corruptione di Aristotele, in AA.VV., Aristotele. Perché la metafisica. Studi su alcuni concetti-chiave della "filosofia prima" aristotelica e sulla storia dei loro influssi, a cura di A. Bausola e G. Reale, Milano, 1994, pp. 377-396, p. 378).

${ }^{3}$ Reale, Introduzione..., p. 73, il quale precisa, però, che la parola 'fisica' non deve portare il lettore moderno a confondere la fisica aristotelica con la fisica moderna. Infatti, quella aristotelica - a differenza della fisica moderna - può definirsi come un "“ontologia' o 'metafisica' del sensibile. Ci troviamo, insomma, di fronte a una considerazione squisitamente filosofica della natura". Migliori (M. Migliori, Ontologia e materia. Un confronto tra il Timeo di Platone e il De generatione et corruptione di Aristotele, in AA. VV., Gigantomachia. Convergenze e divergenze tra Platone e Aristotele, Editrice Morcelliana, Brescia, 2002, pp. 35104, pp. 35-36): "proprio su questo terreno si è realizzata la rottura tra pensiero moderno e concezione classica: ad un'impostazione molto articolata, insieme meccanicistica e finalistica, quantitativa e qualitativa, si è sostituita la trattazione meccanicistica e quantitativa della scienza moderna".

Educ. e Filos. Uberlândia, v. 23, n 45, p. 101-164, jan./jun. 2009. 
del movimento primo che è interno ( $(\dot{\varepsilon} \vee \alpha \hat{\jmath} \tau \hat{\omega})$ in ciascuno degli esseri naturali, in quanto tali $[\ldots]<4>$ Inoltre, si dice physis il principio da cui esiste o si genera un qualche oggetto naturale e che non ha forma $\mathrm{e}$ al quale non è possibile mutare in virtù della propria potenza $[\ldots]<5>$ Inoltre, in un altro senso, si dice physis la sostanza (ov̉ó́ $\alpha$ ) degli esseri naturali $[\ldots]$ Perciò, in tutte le cose che sono e si generano per natura, anche se è già presente ciò da cui si generano o esistono naturalmente, non diciamo che a loro appartiene la physis, se non hanno la forma e la figura ( composto da entrambe [materia e forma]: per esempio gli animali e le loro parti. E la physis che è materia prima [...], è anche $<6>$ la forma $\mathrm{e}$

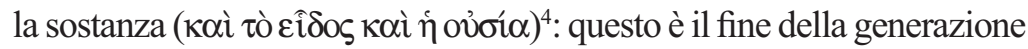
$<7>$ Per estensione, dunque, e in senso generale, ogni sostanza viene detta physis in virtù di questa [della forma], perché anche la forma (ov̉oí $\alpha$ ) è una certa physis. Dalle cose dette risulta che la physis, nel suo senso primo e proprio, è la sostanza delle cose che hanno in se stesse, in quanto tali, il principio di movimento (1014 b 16 - 1015 a 15) .

I primi due significati sono anteriori agli altri anche dal punto di vista etimologico e storico, perché connettono la physis al verbo

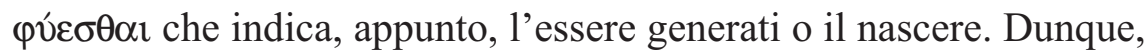
Aristotele si riferisce al "concetto originario di physis proprio dei presocratici, quando afferma che il termine, se inteso con la $y$ lunga, deriva dal verbo phyesthai $[\ldots]$ e perciò indica 'la generazione delle cose che sono generate', oppure significa 'il principio immanente da cui si genera ciò che è generato" "'. L'ultima accezione, invece, come

\footnotetext{
${ }^{4}$ Si noti qui l'uso di ov̉oí $\alpha$ in senso forte per indicare la forma come viene chiarito dal contesto e dalla contrapposizione alla materia. Lo stesso uso del termine occorre alla linea 1015 a 13.

${ }^{5}$ La traduzione di tutti i passi citati è mia.

${ }^{6}$ E. Berti, Nuovi studi aristotelici. Fisica, antropologia e metafisica, volume II, Morcelliana, Brescia, 2005, p. 73, il quale osserva, inoltre, che "qui si allude a due diversi aspetti della natura, quello per cui essa indica il processo del
} 
indica lo stesso Aristotele, è un'estensione, quindi un uso traslato, del sesto significato: ogni sostanza può dirsi natura, in virtù del fatto che la forma è uno dei sensi in cui si dice physis.

Restano, dunque, quattro significati che sembrano i più propri e fondamentali per la concezione aristotelica della la natura:

1. natura in un senso $<3>$ è principio del movimento che è in ciascuno degli esseri naturali. Alla fine del passaggio citato, Aristotele precisa anche che questo è il senso proprio di physis;

2. il primo significato viene legato strettamente al quinto: infatti, nelle linee finali si afferma che physis è la sostanza delle cose che posseggono il principio di movimento in sé stesse. Dunque, potrebbe definirsi come il sinolo, cui, Aristotele accenna chiaramente a 1015 a 6 quando afferma che physis è la sostanza degli esseri naturali, dunque è naturale ciò che è composto da materia e forma.

Infatti,

3. natura in un altro senso ancora è il principio materiale originario che è privo di forma, cioè la materia prima $<$ punto 4, ribadito a 1015 a $7>^{7}$

4. natura è, infine, la forma $<6>$ che Aristotele menziona già a 1015 a 5 parlando della physis come sinolo.

nascere, ovvero del venire all'essere, e quello per cui essa indica il principio da cui le cose nascono, cioè la fonte, l'origine delle cose. E' probabile che presso i presocratici i due aspetti coincidessero. In ogni caso si tratta di un principio globale, di carattere più metafisico che fisico, da cui tutto deriva: è la famosa ó $\rho \chi \eta ́$ o "principio"” (p. 74). Su questa linea, Naddaf (G. Naddaf, Plato and the Пвpì

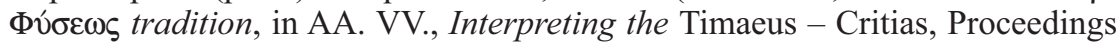
of the IV Symposium Platonicum, Edited by T. Calvo, L. Brisson, Academia Verlag, Sankt Augustin, 1997, p. 27-36, p. 28) sostiene che "i Presocratici nelle loro opere $\pi \varepsilon p \grave{~} \varphi v ́ \sigma \varepsilon \omega \varsigma$ cercavano di spiegare come fosse stato stabilito l'ordine presente delle cose".

${ }^{7}$ Aristotele ne tratta estesamente in De generatione et corruptione, A 1-3. 
Che questi siano gli aspetti che più interessano lo Stagirita nella sua definizione di physis è confermato dal fatto che tale caratterizzazione ritorna con esattezza nel contesto, più proprio e più tecnico, della Fisica aristotelica ${ }^{8}$.

Infatti, nel secondo libro di questo trattato, Aristotele delinea così i confini del terreno fisico:

tra le cose che esistono, alcune sono per natura, altre per altre cause. Sono per natura gli animali e le loro parti e le piante e i corpi semplici, come terra, fuoco, aria e acqua; infatti, diciamo che sono per natura queste e simile cose, cioè tutte quelle cose che appaiono diverse da quelle che non esistono per natura. Infatti, ciascuna di queste cose ha in se stessa il principio del movimento

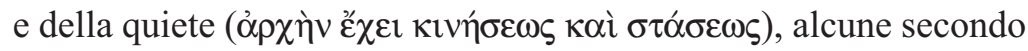
il luogo, altre secondo l'accrescimento e la diminuzione, altre secondo l'alterazione (B, 1, 192 b 8-15).

Innanzitutto, si ribadisce, dunque, l'importanza del movimento, o meglio di un principio interno di movimento, per definire ciò che è naturale.

Poi, nel corso del primo capitolo del libro B, Aristotele ripropone anche $\mathrm{i}$ due sensi di materia e forma, che anche in questo contesto vengono comunque connessi strettamente all'idea di natura quale principio di movimento.

$<1>$ In un senso, pertanto, la natura si definisce così: la materia

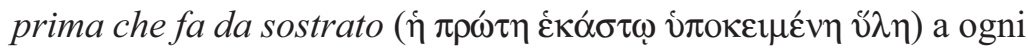
cosa tra quelle che hanno in se stesse il principio del movimento

\footnotetext{
${ }^{8}$ Mansion (Introduction..., p. 94) osserva che qui "Aristotele tenta di stabilire la definizione della natura ponendosi in modo netto da un punto di vista fisico e fondandosi sull'esperienza". A me sembra, invece, che la conclusione sia identica a quella raggiunta nella Metafisica, l'unica particolarità è la distinzione delle diverse forme di movimento.
}

Educ. e Filos. Uberlândia, v. 23, n 45, p. 101-164, jan./jun. 2009. 
e del cambiamento, ma $<2>$ in un altro senso essa è definita

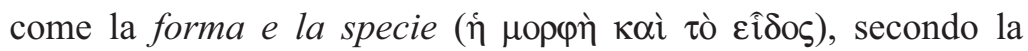
definizione. Come, infatti, si dice arte ciò che è in relazione all'arte e all'artistico, così anche si dice natura ciò che è in relazione alla natura e al naturale [...] Così che, nel secondo senso, la natura delle cose che hanno in se stesse il principio di movimento, potrebbe

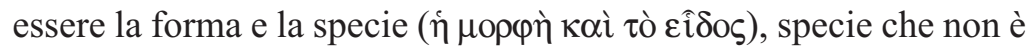

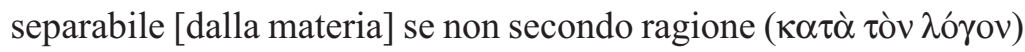
(B, 1, 193 a 28 - 193 b 5).

Prescindendo, per ora, dal riferimento alla possibilità di separazione con un'operazione razionale (questione sulla quale torneremo), da questo passaggio si chiarisce, dunque, che il senso più proprio di physis, nel contesto di quella che Aristotele definisce "scienza della natura ( 16), è principalmente la natura intesa come principio interno di movimento 9 , perché è essenzialmente questo che distingue gli enti naturali da quelli che non lo sono. Tale natura, poi, in un senso può intendersi come la materia di ciò che ha in se stesso tale principio di movimento, in un altro come la forma o la specie. La natura come sinolo di materia e forma - significato che in questo contesto non viene chiaramente distinto - è comunque presente nel momento in cui Aristotele indica come naturali le piante e gli animali; tale significato viene implicitamente ricordato anche nelle righe finali del passo appena citato, perché Aristotele afferma che materia e forma non possono essere separate, se non con il pensiero.

\footnotetext{
${ }^{9}$ A conferma di questo, il terzo libro della Fisica, nel quale Aristotele apre effettivamente l'analisi fisica, tratta proprio del movimento e di tutti i concetti ad esso connessi. Tale principio di movimento si esplica in maniera diversa nelle diverse realtà sensibili: se per gli esseri dotati di anima, tale principio si identifica con l'anima (cfr. De anima, A, 1, 402 a 5-7), per altri enti naturali, ma non animati - come i quattro elementi - il principio che li fa muovere di un moto naturale è identificabile nel pesante e nel leggero. Infatti, Aristotele definisce queste proprietà come "potenze degli elementi" che, realizzandosi, determinano i movimenti degli elementi (cfr. De caelo, $\Delta, 3$ e Fisica $\Theta$, 4).
} 
Dando alla natura questo duplice valore di materia e di forma, Aristotele

pretende allo stesso tempo di riallacciarsi alla dottrina filosofica anteriore e di superarla. Nella sistematizzazione che egli opera della dottrina dei predecessori, quello che essi riconoscono come natura, egli lo pone nella classe della materia. Ma il loro punto di vista è incompleto: la natura in senso proprio è la forma. ${ }^{10}$

Infatti, pur dichiarando tale ambivalenza del concetto di physis, lo Stagirita stabilisce anche un primato della forma sulla materia, forma che, però, come avremo modo di approfondire, va studiata dal physikos sempre in connessione con la materia, mai in se stessa ${ }^{11}$.

E questa (la forma) è natura più di quanto lo sia la materia: ciascuna cosa, infatti, si dice che è allora, cioè quando è in atto, più che quando è in potenza (193 b 6-7).

In piena coerenza con quest'ultima affermazione, Aristotele può definire ancora meglio il campo della natura e delle cose naturali riconoscendo che

sono secondo natura le cose che, mosse continuamente da un qualche principio che hanno in se stesse, giungono ad un qualche fine (199 b 15-17).

\footnotetext{
${ }^{10}$ Mansion, Introduction..., p. 101.

${ }^{11}$ La definizione appena tratteggiata di physis e, quindi, di scienza fisica, trova una netta rispondenza nella descrizione che Aristotele dà della scienza fisica in Metafisica, 七, 1: "Dal momento che, anche la scienza fisica si trova a trattare di un certo genere di essere (tratta, infatti, di una sostanza tale da avere in sé stessa il principio del movimento e della quiete) è chiaro che non è né scienza pratica né poietica" (1025 b 18-21). Dunque, "la fisica sarà una conoscenza teoretica, ma conoscenza teoretica di un genere di essere che ha la potenza di muoversi e della

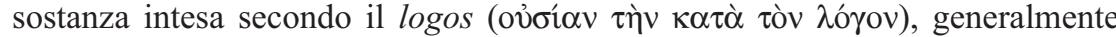

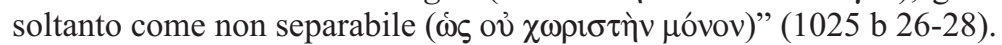


Ora, se questa è la definizione più tecnica e, dunque, il senso più proprio nel quale Aristotele concepisce la natura nei trattati propriamente fisici, è evidente che quella che egli definisce come "scienza o filosofia della natura" abbraccia un terreno molto vasto tale che "non c'è alcun fenomeno propriamente detto che, visto in un determinato modo, non sia di sua competenza". ${ }^{12}$ Infatti, "se l'oggetto è quel genere di sostanza che si muove, potremmo semplificare dicendo che la fisica si occupa di tutte le realtà, escluse quelle metafisiche". ${ }^{13}$

Una prova evidente di tale concezione estesa della scienza della natura si ottiene prendendo in considerazione gli incipit delle opere maggiori che ricadono sotto tale ambito. Nel De caelo, per

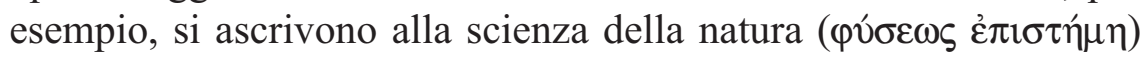
i corpi e le grandezze, le loro affezioni e i loro movimenti, nonché i principi di tale sostanze (De caelo, A, 268 a) e, così facendo, si fa ricadere nello stesso studio fisico tanto ciò che oggi si attribuirebbe a uno studio più propriamente astronomico - il kosmos, gli astri e le stelle (libri I e II) ${ }^{14}$ - quanto la ricerca intorno agli elementi del cosiddetto mondo sublunare (libri III e IV). ${ }^{15}$ Analogamente, il De

${ }_{12}$ Mansion, Introduction..., p. 38.

${ }^{13}$ Migliori, Ontologia ..., p. 36, corsivo mio.

${ }^{14}$ A conferma che per Aristotele 1'astronomia non è che una parte della fisica stessa, cfr. anche Fisica, B, 2: 'Inoltre, 'si deve esaminare se' l'astronomia sia una scienza diversa oppure una parte della fisica: se, infatti, spetta al fisico sapere che cosa sono il sole e la luna, è assurdo, poi, che non conosca nulla circa i loro attributi particolari, inoltre anche perché è evidente che coloro che trattano della natura e della figura della luna e del sole, 'trattano anche la questione' se la terra e il cosmo siano sferici o meno" (193 b 26-30). A questo proposito, Mansion (Introduction..., p. 187) osserva infatti che "quando si legge il [...] De caelo, uno scritto fisico, indubbiamente, i due primi libri sono pieni di discussioni relative agli astri".

15 In questo senso, non sembra esatto quanto afferma Wieland (W. Wieland, Die aristotelische Physik. Untersuchungen über die Grundlegung der Naturwissenschaft und die sprachlichen Bedingungen der Prinzipienforschung bei Aristoteles, Vandenhoeck \& Ruprecht, 1970; traduzione italiana di C. Gentili,

Educ. e Filos. Uberlândia, v. 23, n 45, p. 101-164, jan./jun. 2009. 
generatione et corruptione si presenta come un'indagine intorno alla generazione e alla corruzione "di ciò che per natura si genera e si corrompe" (A, 1, 314 a 1-2) e offre una descrizione dell'intero mondo naturale. Dunque, "questa fisica aristotelica sembra innanzitutto una metafisica dei corpi o una filosofia del mondo materiale"16 il cui ampio spettro viene del resto confermato dalle parole dello stesso Aristotele nell'incipit dei Meteorologia, dove egli sembra tracciare un percorso logico dell'indagine fisica. ${ }^{17}$

La Fisica di Aristotele. Studi sulla fondazione della scienza della natura e sui fondamenti linguistici della ricerca dei principi in Aristotele, Laterza, Bologna, 1993, p. 21), quando osserva che "per Aristotele, la cosmologia è qualcosa di diverso dalla fisica. La cosmologia, così come si presenta nei quattro libri del De caelo e negli scritti ad essi successivi, è una dottrina che si occupa delle cose naturali e delle loro proprietà, e di quella cosa in primo luogo che nella gerarchia delle sostanze naturali si colloca al posto più alto: il cielo. Al contrario, la fisica non si interroga in genere su determinate cose naturali, ma sulle forme fenomeniche universali e sui principi delle cose naturali". Ora, come si è cercato di mostrare, i testi aristotelici non fanno questa distinzione, ma ascrivono alla physis e, dunque, anche alla scienza della natura, tutte le cose naturali. Certo, il trattato di Fisica - che, come vedremo immediatamente, ricopre un ruolo logico di prologo ai vari trattati di fisica - si occupa dei principi di tale scienza e, in questo senso si distingue dal De caelo, ma questa è cosa diversa da quanto qui afferma lo studioso tedesco.

${ }^{16}$ Mansion, Introduction..., p. 38. Così anche (C. Kahn, La Physique d'Aristote et la tradition greque de la philosophie naturelle, in AA. VV., La Physique d'Aristote e les conditions d'une science de la nature, Actes du Colloque organisé par le Séminaire d'Epistémologie et d'Histoire des Sciences de Nice, Edités par F. De Gandt et P. Souffrin, Librairie Philosophique J. Vrin, Paris, 1991, pp. 41-52, pp. 41-44; il quale evidenzia che "Aristotele [...] concepisce il dominio studiato dal physikos come lo stesso, essenzialmente, del racconto di Socrate" nel Fedone (96 A-B) in cui Platone presenta le questioni inerenti allo studio peri physeos coprendo un'ampiezza confrontabile: "ovviamente la struttura del mondo e i fenomeni del cielo, ma anche le origini della vita e della natura del pensiero umano".

${ }^{17}$ A proposito di questo passo, Pepe (Aristotele, Meteorologia, Introduzione, traduzione, note e apparati di L. Pepe, Bompiani, Milano, 2003, p. 27, n. 2) sostiene che sia innegabile che "il proemio, nel quale sono riassunti in forma sistematica questi studi, dia l'impressione di un ordinamento a-posteriori di lavori che quasi certamente avevano avuto un andamento meno lineare". Solmsen

Educ. e Filos. Uberlândia, v. 23, n 45, p. 101-164, jan./jun. 2009. 
Dunque, si è parlato in precedenza delle cause prime della natura e di ogni movimento naturale; inoltre, degli astri ordinati secondo la traslazione superiore e degli elementi dei corpi, quali e quanti siano, e del loro mutamento reciproco e della generazione e corruzione in generale. E' rimasta ancora da esaminare la parte di questa ricerca che tutti i predecessori chiamavano meteorologia (A, 1, 338 a 20-26).

\section{La fisica dei corpi viventi}

Nell'orizzonte allargato di questa physis, intesa come principio di movimento, trovano cittadinanza legittima, come si è appena visto,

(F. Solmsen, Aristotle's System of the Physical World, Cornell University Press, Ithaca, New York, 1960, p. 406) ipotizza, invece, che Aristotele, con questo trattato, intendesse colmare una mancanza nel proprio sistema fisico e questo proemio lo dimostrerebbe. Migliori (M. Migliori, Aristotele, La generazione e la corruzione, traduzione, introduzione e commento di M. Migliori, Loffredo Editore, Napoli, 1976, pp. 23-24) nota che, comunque, il catalogo di Andronico - in cui le opere fisiche appaiono in quest'ordine: Fisica, De caelo, De generatione et corruptione, Meteorologica - trova conferma in questo proemio, il che fa pensare che sia possibile "inquadrare la logica che sottende lo sviluppo delle opere fisiche", precisando, però, che si è di fronte ad uno schema logico e, dunque, "non si vuole affatto affermare che queste opere siano state concepite cosi". Del resto "che i trattati precedenti siano stati redatti secondo questo piano e in vista di realizzarlo o no, non importa. E' chiaro che l'autore ora vuole farceli entrare e che ne parla come di una parte già realizzata" (Mansion, Introduction..., p. 17). A questo proposito, poi, è importante ricordare che, com'è noto, il Corpus Aristotelicum "è costituito non dalle opere scritte e destinate alla pubblicazione (che sono andate perdute pressoché per intero), ma dalle opere di scuola, le quali erano destinate solo all'attività didattica e di ricerca". E' chiaro, dunque, che non si ha a che fare con dei libri, ma con veri e propri "corsi di lezione" che, in quanto tali, costituiscono "sempre un materiale non solo in continuo fieri, ma mai definitivamente ultimato [...], ma c'è di più. Molte delle opere aristoteliche, così come oggi le leggiamo, sono nate dalla fusione di vari corsi di lezioni (di vari "libri"), operata in parte da Aristotele stesso, in parte dai suoi discepoli e in particolar modo da Andronico di Rodi" (G. Reale, Aristotele, Metafisica, a cura di G. Reale, Rusconi, Milano, 1993, p. VI dell'introduzione). Ora, l'incipit dei Meteorologia sembra dare proprio testimonianza di questo, perché questo elenco di argomenti trattati e da trattare si giustifica pienamente in un contesto didattico. A proposito di questo passo, cfr. anche J. Brunschwig, Qu'est ce que "la physique" d'Aristote?, in AA. VV., Physique..., pp. 11-37, pp. 25-27. 
anche "gli animali e le loro parti e le piante [...] Infatti, ciascuna di queste cose ha in se stessa il principio del movimento e della quiete" (Fisica, B, 1, 192 b 10-15) ${ }^{18}$. Dunque, è già evidente da queste parole che lo Stagirita - data la sua definizione di "natura" - coinvolge nella fisica anche ciò che oggi comunemente si fa rientrare nel campo più propriamente biologico: gli animali e le piante, ${ }^{19}$ appunto.

La conferma di una tale estensione la si ha dai cosiddetti scritti biologici che lo Stagirita definisce e sente comunque come fisici, senza alcuna soluzione di continuità ${ }^{20}$. Infatti egli afferma che delle questioni che essi pongono, cioè appunto, degli animali e delle loro parti, deve occuparsi il physikos. ${ }^{21}$

\footnotetext{
${ }^{18}$ Risulta del tutto confrontabile, dal punto di vista contenutistico, anche quanto Aristotele afferma nel De caelo, facendo riferimento ad animali e piante come ad oggetti propriamente fisici: "Dal momento che, di ciò che viene detto per natura (

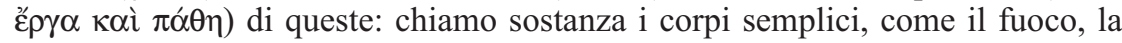
terra e i corpi che ne sono costituiti, come il cielo nel suo complesso e le sue

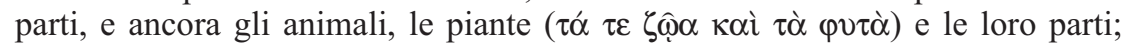
chiamo passioni e attività i movimenti di ciascuno di questi esseri e quelli degli altri esseri, (movimenti) che sono causati dai primi in virtù della loro potenza e, inoltre, le loro alterazioni e i cambiamenti dell'uno nell'altro; è evidente che la maggior parte della ricerca sulla natura interessa i corpi: infatti tutte le sostanze naturali o sono corpi o dipendono dai corpi e dalle grandezze" (De caelo $\Gamma, 1$, 298 a 27-298 b 4).

${ }^{19}$ A questo proposito Hicks (R. D. Hicks, Aristotle, De anima, with translation, introduction and notes by R.D. Hicks, Cambridge University Press, Cambridge, 1907; ristampe Amsterdam, 1965; Hildesheim, 1990, p. XI) nota che "la sua scienza della natura o fisica, è passata dall'inorganico ai bordi della vita organica".

${ }^{20}$ Per questo, a mio parere, non è corretto escluderli dal conteggio dei trattati fisici, come fa Mansion (Introduction..., p. 44), il quale, come si è già accennato, afferma che nell'edizione Bekker "bisogna togliere dal totale lo spazio occupato dagli scritti di un' altra categoria, come l'Historia animalium".

${ }^{21}$ Kahn (C.H. Kahn, Sensation and Consciousness in Aristotle's Psychology, in AA. VV., Articles on Aristotle, edited by J. Barnes, M. Schofield, E. Sorabji, Duckworth, London, 1979, pp. 1-31, p. 4) si pone su questa linea quando afferma: ' $E$ ' in questo contesto biologico piuttosto che strettamente psicologico
} 
Questo ramo della fisica rappresenta certamente uno degli interessi più vivi per Aristotele ${ }^{22}$ e sottintende un difficilissimo argomento, cui in questa sede si può soltanto accennare, cioè la trattazione dell'anima che, come infatti viene chiarito immediatamente all'inizio del De anima, è il principio degli esseri animati. $^{23}$

Poiché consideriamo la conoscenza tra le cose belle e degne di onore e una più di un'altra o per il rigore o perché si occupa di oggetti migliori e più ammirabili, per entrambe queste ragioni potremmo ragionevolmente porre tra le prime la ricerca sull'anima. Sembra anche che la conoscenza dell'anima contribuisca di molto ad ogni verità e soprattutto a quella della natura ( $\mu$ ó $\lambda \iota \sigma \tau \alpha$ de $\pi \rho \grave{s}$

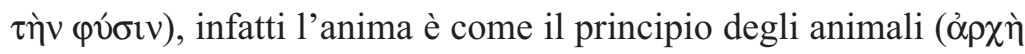
$\tau \hat{\omega} \vee \zeta \omega \omega \nu)$ (De anima, A, 1, 402 a 1-7).

che Aristotele pone la sua dottrina della psyche”. Lo stesso riconosce Lloyd (G.E.R. Lloyd, Aristotle. The Growth and Structure of His Thought, Cambridge University Press, 1968; traduzione italiana di M. Fantuzzi, Aristotele. Sviluppo e struttura del suo pensiero, Il Mulino, Bologna, 1985, p. 129) quando afferma

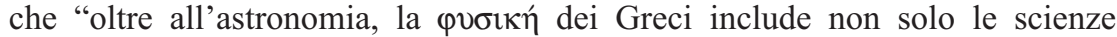
che chiameremmo dinamica, fisica e chimica, ma anche tutti i vari rami della biologia".

22 "Aristotele ha scritto di biologia più che di ogni altra scienza e ha raccolto un vasto dossier di materiale sulle peculiarità (e le somiglianze) delle diverse specie animali, riflettendo anche teoreticamente sui modi appropriati di classificarli per riuscire meglio a spiegare la loro struttura. E queste spiegazioni sono per Aristotele almeno in parte teleologiche" (R. J. Hankinson, Science, in AA. VV., The Cambridge Companion to Aristotle, edited by J. Barnes, Cambridge University Press, 1995, pp. 140-165, p. 158).

${ }^{23}$ Proprio in virtù di tale definizione dell'anima si può a buon diritto ritenere che, come afferma Movia (Aristotele, L'anima, a cura di G. Movia, Loffredo Editore, Napoli, 1979, p. 34), “"nell'ordinamento del corso biologico di Aristotele, il nostro trattato occupa un posto centrale e che, da un punto di vista logico, esso ne costituisce la "prefazione generale"'. Si riconosce, dunque, al De anima nell' ambito delle opere che definiremmo biologiche - un ruolo analogo a quello della Fisica nell'ambito delle opere per noi più propriamente fisiche.

Educ. e Filos. Uberlândia, v. 23, n 45, p. 101-164, jan./jun. 2009. 
Un'ulteriore conferma, in questo senso, ci viene dal De partibus animalium dove, sulla stessa linea del De anima, Aristotele torna a ribadire che, dato che in ciò che è animato è evidentemente implicata anche l'anima, il fisico deve occuparsene.

Se poi questo è l'anima o una parte dell'anima o non può essere senza l'anima (effettivamente, se se ne va, non c'è più l'animale vivente, né alcuna delle sue parti rimane la stessa, tranne la sola forma esteriore, come le creature mitiche che sono pietrificate), se dunque le cose

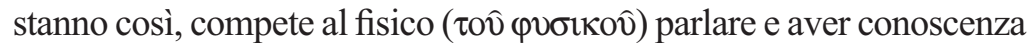
dell'anima, se non nella sua totalità, almeno per quanto in essa fa sì che l'animale sia tale, e dire che cosa è l'anima o proprio questa parte e parlare degli attributi secondo questa sua essenza: del resto, anche natura si dice in due sensi, che sono natura come materia e natura come forma (ov̉ó́as). ${ }^{24} \mathrm{Ed}$ è questa anche come ciò che muove e come il fine. Tale è l'anima dell'animale o nella sua totalità o una qualche sua parte. Cosicché, anche da questo punto di vista, chi studia la natura dovrebbe parlare dell'anima piuttosto che della materia, quanto più in considerazione del fatto che la materia è natura a causa di questa, piuttosto che il contrario (e infatti il legno è letto e tripode, perché è queste cose in potenza) (A, 1, 641 a 17-32).

Prescindendo dal problema esplicitamente posto in queste righe e cioè se il naturalista debba occuparsi di tutta l'anima o solo di una parte, è evidente da questi passi che Aristotele non vede alcuna frattura tra quella che noi chiamiamo fisica e la biologia, cioè la fisica dei corpi viventi in cui, appunto, diventa fondamentale il concetto di anima: tutto rientra nel campo d'indagine del physikos. ${ }^{25}$

${ }^{24}$ Anche qui, come in Metafisica, $\Delta, 4,1015$ a 13, ov̉oí $\alpha$ è usato nel senso forte di forma, in questo caso, contrapposta alla materia (cfr. n. 4, p. ***).

${ }^{25}$ Kahn (Physique..., p. 44), a questo proposito, nota, infatti, che "i testi aristotelici di psicologia e fisiologia (De anima e Parva Naturalia) così come i grandi trattati di biologia, sono tutti concepiti come facenti parte di questa impresa globale che 


\section{Il physikos come studioso della forma e della materia}

L'individuazione del terreno nel quale opera il physikos è un problema che Aristotele affronta con argomentazioni ad hoc nella Fisica e nel De anima, attraverso un confronto tra questa figura e, rispettivamente, quella del matematico e del dialettico.

Nel secondo libro della Fisica, egli afferma che bisogna osservare in che cosa il matematico differisca dal fisico. Infatti, anche i corpi fisici hanno superficie, volume, lunghezza e punti, cioè tutte quelle realtà di cui si occupa il matematico (B, 2, 193 b), ma $d a$ un punto di vista diverso.

Infatti, quest'ultimo se ne occupa

a) non in quanto ciascuna di esse sia il limite di un corpo fisico;

b) non studia gli attributi, in quanto attributi di un corpo fisico.

Il matematico applica, infatti, una separazione, nell'oggetto del suo studio, tramite un'operazione di sottrazione o astrazione ( $\alpha \alpha^{\prime} \hat{i}^{\prime}$

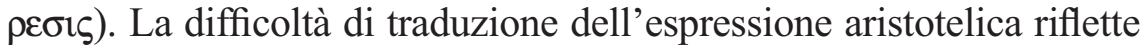
una situazione problematica nella definizione dell'operazione stessa, ma è chiaro che tale procedimento permette al matematico di studiare, per esempio, la linea o la superficie, non in quanto linea e superficie di un dato corpo fisico, ma in se stesse ${ }^{26}$. Questo comporta che il

\footnotetext{
è lo studio della natura".

${ }^{26}$ La Cattanei (E. Cattanei, Enti matematici e metafisica. Platone, l'Accademia e Aristotele a confronto, Vita e pensiero, Milano, 1996, p. 202) richiama l'attenzione sulla traduzione letterale del termine ó $\varphi \alpha i ́ p \varepsilon \sigma ı \varsigma$ perché riconosce che il fatto di tradurlo con "astrazione" "crea la difficoltà di stabilire che tipo di attività mentale sia l'astrazione secondo Aristotele, che tipo di implicazioni ontologiche abbia e se riesca davvero a spiegare l'oggetto e la verità delle scienze matematiche. Ma le intenzioni di Aristotele non potrebbero essere più modeste? La 'sottrazione' è, per definizione, qualcosa di puramente logico, relativo a 'nozioni', qualcosa di appartenente all'ordine del conoscere, che non ha automatiche e necessarie ripercussioni nell'ambito dell'essere e dell'ontologia". La studiosa ipotizza dunque che Aristotele con questo termine voglia semplicemente intendere che "i matematici lavorano su nozioni ottenute 'per sottrazione' da nozioni più complesse". Mansion, (Introduction..., p. 134; p.
}

Educ. e Filos. Uberlândia, v. 23, n 45, p. 101-164, jan./jun. 2009. 
matematico possa prescindere dal movimento che, invece, come si è visto, è l'elemento più propriamente definitorio della physis.

Infatti, con il pensiero, essi sono separabili dal movimento

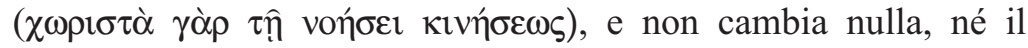
separare comporta errore (B, 2, 193 b 34-35) $)^{27}$.

La possibilità di astrazione è il primo e forse il più importante punto di distanza che Aristotele individua tra il matematico e il fisico, tant'è vero che lo Stagirita insiste su questo punto affermando che si giungerebbe ad una chiarificazione, su questo tema, se si cercasse di

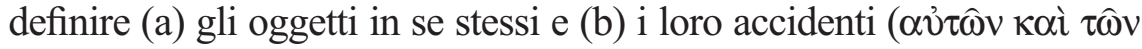
$\left.\sigma \nu \mu \beta \varepsilon \beta \eta \kappa \sigma_{\tau} \omega \nu\right)$.

138) individua, invece, nell'elemento aristotelico della sottrazione o astrazione una vera e propria "teoria dell'astrazione" che legge come "una reazione contro l'intellettualismo realista di Platone [...]. Per Aristotele gli intelligibili sono degli astratti, astratti innanzitutto di cose materiali, percepiti tramite i sensi"; le scienze teoriche sarebbero, dunque, poste in una gerarchia secondo il proprio grado di astrazione. Egli precisa, però, che un grado di astrazione deve porsi anche nella fisica, dal momento che il fisico "non studia 'questa carne qui, quest'osso qui' [...], ma la carne, l'osso". Ora, anche se tale interpretazione appare del tutto logica, bisogna notare che dai testi aristotelici citati, non sembra possibile trarla. Infatti, per lo Stagirita l'alternativa è netta: il matematico opera una separazione nei suoi oggetti tra forma e materia (e in questo senso astrae), mentre il fisico non lo fa. A questo proposito, dunque, sembra esatto quanto osserva Mignucci (M. Mignucci, In margine al concetto di forma nella Metafisica di Aristotele, in AA.VV., Aristotele [...], pp. 145-170, p. 166) quando precisa che "la soluzione di Aristotele non si muove lungo le linee di un approccio psicologico [...]. Il

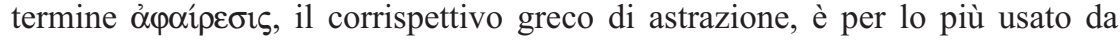
Aristotele con riferimento agli oggetti della matematica. Nel suo linguaggio la linea e il circolo sono il prodotto di un'astrazione, mentre non lo sono l'uomo

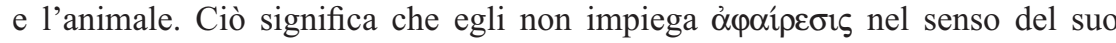
corrispondente moderno".

${ }^{27}$ Incidentalmente, Aristotele osserva che l'operazione del matematico non è erronea a differenza di quanto avviene per i "sostenitori delle Idee" che inconsapevolmente «separano gli oggetti fisici, che pur sono meno separabili

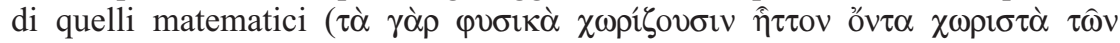
$\mu \alpha \theta \eta \mu \alpha \tau \imath \kappa \hat{\omega} v) »(\mathrm{~B}, 2,193$ b 36-194 a 1). 
Il dispari, il pari, il retto, il curvo e, inoltre, il numero, la linea, la figura sono privi di movimento, ma non lo sono la carne, l'osso, l'uomo, ma tali cose vengono dette, così come naso 'camuso' e non curvo (B, 2, 194 a 3-7).

Dunque, agli oggetti quali la carne, l'osso, l'uomo (di cui si occupa il fisico) è connaturato il movimento (a differenza di quelli di cui si occupa il matematico che ne sono privi, appunto in virtù dell'astrazione cui la matematica sottopone i propri oggetti di studio) ed essi accolgono qualificazioni più "materiali" e non quelle propriamente matematiche. Per esempio, nel caso del naso, il suo accidente sarà di essere camuso e non curvo. Ora, pur essendo questi concetti in qualche misura analoghi, il camuso si distingue dal curvo, proprio perché viene concepito insieme alla materia, mentre il curvo può prescinderne ${ }^{28}$.

E' evidente, infatti, che uno dei tratti che caratterizza lo studio del physikos è proprio questa congiunzione tra forma e materia, dato che, come si è visto, la natura si dice in due modi: in un senso come materia (cfr. Fisica, B, 1, 193 a 27-28); in un altro come forma (cfr. Fisica, B, 1, 193 b 3-5) e, dunque si deve studiarla considerando entrambi gli aspetti. In questo senso, afferma Aristotele, essa va studiata nello stesso modo in cui si studierebbe la camusità, "perché tali cose non sono prive di materia né sono conformi alla materia" (B, 2, 194 a 14-15).

Analogamente, quindi, anche la natura è qualcosa di non privo di materia, ma non è esclusivamente materiale e, per coglierla, non si può astrarre dalla materia (come fa il matematico), ma neanche limitarsi soltanto ad essa.

Del resto - nota incidentalmente Aristotele - questa differenza

${ }^{28}$ Dunque, come osserva Mansion (Introduction..., p. 140) "per Aristotele [...] gli stessi intelligibili di ordine matematico sono degli astratti, ottenuti tramite astrazione a partire da quei dati soggetti a mutamento e molteplici che sono i sensibili materiali".

Educ. e Filos. Uberlândia, v. 23, n 45, p. 101-164, jan./jun. 2009. 
tra il fisico e il matematico è dimostrata anche da quelle scienze matematiche che si avvicinano in misura maggiore alla natura, come l'ottica, l'armonica, l'astronomia e che, infatti, si trovano in un rapporto antitetico con la geometria:

infatti la geometria prende in esame la linea fisica, ma non in quanto fisica; l'ottica, invece, la linea matematica, ma non in quanto matematica, ma in quanto fisica (B, 2, 194 a 9-12).

Stando così le cose, Aristotele sostiene che il fisico dovrebbe condurre la propria indagine «su ciò che risulta da entrambe», cioè dalla materia unita alla forma (B, 2, 194 a 16-18).

Dunque, il fisico, a differenza del matematico, studia gli oggetti naturali senza operare alcuna separazione tramite astrazione. E' chiaro allora che egli deve indagare tanto sulla forma quanto sulla materia, o meglio sul sinolo di forma e materia. A questo punto, però, Aristotele si chiede se la conoscenza di queste due realtà sia compito di una sola e medesima scienza o se si debba far ricorso a un'altra scienza, per ribadire che è proprio della fisica occuparsi di entrambe.

Se l'arte imita la natura e compete alla stessa scienza conoscere

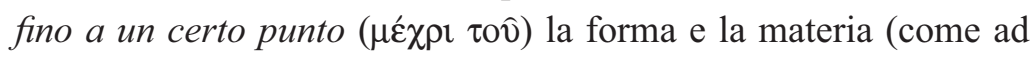
esempio compete al medico conoscere la salute e la bile e il muco, cose nelle quali si trova la salute e, allo stesso modo, compete al costruttore conoscere la forma della casa e la materia, cioè mattoni e legna e così per quelli che praticano altre arti), anche alla fisica può competere di conoscere entrambe le nature (B, 2, 194 a 21-27).

La precisazione secondo cui la forma deve essere conosciuta "fino a un certo punto" sembra circoscrivere e precisare ulteriormente il terreno della fisica rispetto alla metafisica. Infatti, lo studio della forma - proprio in virtù del fatto che non si può fare astrazione dalla 
materia - risulta limitato. Il fisico, cioè, non studia la forma in se stessa. $^{29}$

\section{Il physikos come studioso del corpo vivente}

Una trattazione sotto certi aspetti analoga - e forse ancora più ricca - è ricavabile dal De anima. Infatti, dopo aver elencato i problemi legati alle passioni dell'anima (A, 1, 403 a), Aristotele apre una parentesi metodologica dicendo che è evidente che queste

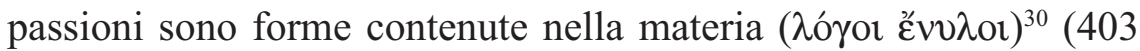
a 25); per questo è compito del fisico trattare dell'anima, perché, appunto, anche in questo caso, egli ha a che fare con un composto di materia e forma.

Ora, in questo contesto, Aristotele traccia la differenza che corre tra il fisico e il dialettico, con una movenza del tutto confrontabile a quella verificata nella Fisica rispetto al matematico. Si afferma, infatti, che fisico e dialettico definiscono le stesse cose, ma in maniera diversa. Per esempio, la collera dal fisico è descritta come un'ebollizione del sangue e del calore intorno al cuore, dal dialettico come il desiderio di far male a propria volta. Dunque, il fisico guarda alla materia ( $(\check{\lambda})$ ), il dialettico alla forma e all'essenza

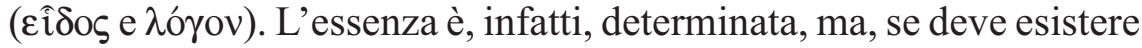
in atto, ha bisogno di una materia. In modo analogo, la casa può essere descritta come un riparo o come fatta di pietra e di legno.

Esattamente come in Fisica, B, Aristotele a questo punto, si

\footnotetext{
29 'Lo studio della forma e dell'essenza nella fisica si distingue nettamente dallo stesso studio nella metafisica. Questo deve esaminare, infatti, il caso delle essenze che non hanno alcun rapporto con la materia" (Mansion, Introduction..., p. 205)

${ }^{30}$ La conferma e l'esplicitazione di questa espressione si ha alla fine del capitolo quando Aristotele ribadisce che «le passioni dell'anima sono, così, inseparabili $(\hat{\alpha} \chi \omega \hat{\omega} \iota \sigma \tau \alpha)$ dalla materia fisica degli animali, in quanto proprio tali passioni sono, per esempio, l'ira e la paura e non come la linea e la superficie» (A, 1, 403 b 17-19).
}

Educ. e Filos. Uberlândia, v. 23, n 45, p. 101-164, jan./jun. 2009. 
chiede se il fisico sia

- quello che considera solo la forma, ma è chiaro che la risposta è negativa, perché questi è, appunto, il dialettico;

- chi considera solo la materia senza forma; questo sembrava risultare dalla linea di demarcazione tracciata rispetto al dialettico, anche se si è già adombrata l'esistenza del composto di forma e materia facendo riferimento alle passioni dell'anima;

- se non sia, invece, chi le considera entrambe (A, 1, 403 b).

In piena coerenza con quanto affermato in Fisica, la scelta di Aristotele va a quest'ultima alternativa; egli spiega anche come si debbano intendere le altre figure cui si fa implicito riferimento nell'elenco appena esposto, ampliando ulteriormente il quadro.

Infatti, lo Stagirita distingue due figure che si occupano delle proprietà inseparabili della materia e non le considerano, dunque, in quanto separabili:

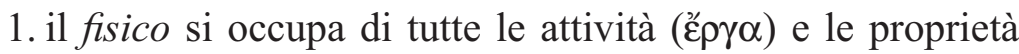
( $\pi \alpha \dot{\theta} \theta \eta)$ di un determinato corpo e di una determinata materia. Quindi, il fisico si occupa del corpo che ha in se stesso un principio di movimento, considerando inseparabili il corpo e le sue caratteristiche;

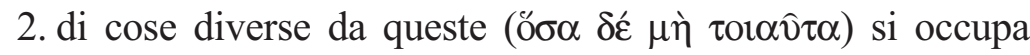
il tecnico ( $\tau \varepsilon \chi v i ́ \tau \eta \zeta)$, la cui figura varia secondo i casi, per esempio l'architetto o il medico. Certo, la delimitazione del terreno del tecnico in questo contesto risulta quanto mai vaga, ma almeno Aristotele lo accomuna al fisico per il fatto che anch'egli considera le proprietà inseparabili dei corpi.

Infine, lo Stagirita elenca anche coloro che studiano tali caratteristiche, ma considerandole separate dai corpi. Anche in questo caso, distingue due figure: 
1. il matematico che studia le caratteristiche che in se stesse non sono separabili, ma non vengono considerate come affezioni di un determinato corpo e sono ottenute per astrazione. Dunque - analogamente a quanto affermato in Fisica, B - il matematico si occupa di caratteristiche che non sono separabili, separandole dal corpo per astrazione, diversamente dal fisico che, invece, le considera insieme al corpo;

2. infine, le caratteristiche dei corpi considerate in quanto separate, sono oggetto dello studio del filosofo primo ${ }^{31}$.

${ }^{31}$ Rorty (A.O. Rorty, De anima: its agenda and its recent interpreters, in M.C. Nussbaum - .O. Rorty (eds), Essays on Aristotle's De anima, Clarendon Press, Oxford, 1992, pp. 7-13, p. 8), a mio parere, offre una lettura non del tutto esatta di questo passaggio. Sostiene, infatti che le affezioni "coinvolgono sia la cognizione sia il corpo (meta sōmatos; 403 a 25); perciò, per spiegare le affezioni dell'anima (pathe $)$ è necessaria la cooperazione di due specialisti': il fisico dà un'analisi della condizione fisica del corpo, ma egli deve assorbire il lavoro del dialettico che chiarisce le cognizioni centrali che sono costitutivamente associate ad ogni affezione. Dunque, in un senso i resoconti fisici e cognitivi sono separabili, in un altro senso, non lo sono: infatti, che essi siano separabili nel pensiero è evidente dal fatto che sono soggetti di due distinti tipi di inchiesta, una fisiologica e l'altra cognitiva. Che essi non siano di fatto separabili, è evidente dall'affermazione di Aristotele secondo cui le spiegazioni riguardanti le affezioni dell'anima sono incomplete se non si considerano entrambi i resoconti. A mio parere, in quest'analisi, condivisibile nel ragionamento, lo studioso non coglie esattamente l'intento aristotelico, nel momento in cui distingue nettamente due figure di specialisti e sembra attribuire al fisico soltanto l'ambito materiale, mentre al dialettico quello cognitivo. Infatti, il testo chiarisce che si definisce fisico non colui che si interessa solo dell'aspetto materiale, ma colui che, di fronte ad un particolare oggetto della fisica, qual è il corpo animato, consideri tanto il corpo quanto le sue affezioni, perché appunto esse sono inseparabili. Si dice, infatti, che non si darebbe una buona definizione della rabbia o della casa (dunque, non sarebbe buona neanche dal punto di vista fisico) se ci si limitasse a dire che la rabbia è ebollizione del sangue e la casa è un insieme di materiali. 


\section{Un approccio gnoseologico: le diverse prospettive possibili per capire la realtà}

Il quadro fin qui ricostruito rende evidente la vastità del terreno nel quale il physikos deve muoversi e l'estrema complessità del suo oggetto di indagine, dato che lo studioso della natura deve occuparsi di una forma sempre congiunta alla materia e al movimento, nell'ambito di una scienza che Aristotele definisce come episteme di "ciò che accade sempre o per lo più" (Fisica, B, 8, 198 b 35). "La fisica è infatti scienza del divenire: essa documenta che si può ragionare con una certa necessità intorno a ciò che muta. In Aristotele, la formula in genere o per lo più assume l'andatura del mondo - la media vita - come prima evidenza". ${ }^{32}$

Ora, di fronte a una realtà che si presenta complessa e multiforme, è possibile verificare, in tutto il pensiero aristotelico (non solo nel contesto fisico), un approccio gnoseologico di fondo che consiste nel mettere in gioco molteplici schemi interpretativi per spiegare una medesima realtà. Infatti, "l'affermazione della polisensicità dei termini da una parte, la dialettica platonica dall'altra, hanno comportato la scoperta che una stessa realtà è sempre analizzabile da diversi punti di vista, legittimi e utili che devono essere rispettati nella loro irriducibilità". ${ }^{33}$ Tale convinzione spinge Aristotele a moltiplicare gli schemi interpretativi della realtà, per cui, di fronte ad un fenomeno da spiegare,

1. in un senso è vera una cosa, ma in un altro senso è vera anche un'altra e diversa cosa;

2. la medesima realtà può essere considerata da punti di vista diversi e, dunque, comportare conclusioni diverse, ma non contraddittorie.

\footnotetext{
${ }^{32} \mathrm{~S}$. Natoli, Aristotele e la scientificità della filosofia. In genere e per lo più (ỏ

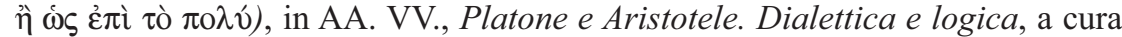
di M. Migliori - A. Fermani, Morcelliana, Brescia, 2008, pp. 295-321, p. 302.

${ }^{33}$ Migliori, Ontologia ..., p. 102.
} 
Le opere fisiche aristoteliche ci offrono diverse occasioni nelle quali verificare queste movenze esplicative funzionali a seguire e giustificare le pieghe del reale senza semplificarle, ma anzi moltiplicando i punti di osservazione.

Un esempio particolarmente significativo della polisensicità si rintraccia in Fisica, $\Gamma$, nel contesto della trattazione di un concetto problematico come l'infinito. Tale concetto, fondamentale per il physikos, per alcune ragioni sembra non esistere, ma, d'altro canto, è impossibile che non esista. Dunque, Aristotele supera l'aporia affermando che "in un senso l'infinito è, in un altro non è" $(\Gamma, 6,206$ a 14). Infatti, si chiarirà in seguito che l'infinito esiste in potenza per divisione e per aggiunzione, ma non esiste in atto e non esiste come corpo sensibile $(\Gamma, 6,206$ b 12-20).

Del tutto confrontabile a questa è anche la trattazione del luogo, perché anche il luogo può dirsi in due sensi:

dal momento che in un senso si dice di ciò che è per sé ( $\kappa \alpha \theta^{\prime} \alpha$ iò), in un altro ciò che è per altro ( $\left.\kappa \alpha \tau^{\prime}{ }^{\prime}{ }^{\prime} \lambda \lambda \mathrm{o}\right)$, anche il luogo, $<1>$ da una parte, è quello comune in cui si trovano tutti i corpi, $<2>$ dall'altra è quello particolare in cui immediatamente un corpo si trova (209 a 31-33).

Dunque, il luogo per sé è ciò in cui sono tutti i corpi e, per altro è il luogo riferito ad un determinato corpo.

Dico, per esempio: tu ora sei nel cielo, perché sei nell'aria quest'aria, infatti, si trova nel cielo - e sei nell'aria perché sei sulla terra e, allo stesso modo, sei in quest'ultima, perché ti trovi in questo luogo qui che contiene esclusivamente te (209 a 33-b 1).

Il meccanismo descritto potremmo definirlo a scatole cinesi: il luogo per sé è il cielo, il luogo per altro è, invece, il luogo determinato. 
Ora, il luogo inteso in questa seconda accezione si definisce come

un certo limite, cosicché il luogo potrebbe sembrare la conformazione e la forma ( cosa, mediante la quale si limitano la grandezza e la materia della grandezza (209 b 2-4).

Ma, inteso per sé, cioè come ciò in cui si trovano tutti i corpi, esso sembra essere l'intervallo della grandezza e, dunque, può definirsi nei termini di materia, perché

questa, infatti, è diversa dalla grandezza, è ciò che è contenuto e limitato dalla forma, come da un piano e da un limite, e la materia e l'indeterminato sono così: qualora, infatti, vengano sottratte da una sfera il limite e le proprietà, non resterà nulla tranne la materia (209 b 7-10).

Dunque, proprio come l'infinito, il luogo deve esistere, ma è di difficile definizione, perché

$<1>$ in quanto è separabile dalla cosa, esso non ne è la forma; ma $<2>$ in quanto esso contiene la cosa, è diverso dalla materia $(\Delta, 2$, 209 b 32).

Ariprova dellaradicalità e della pervasività ditale atteggiamento, la stessa physis, come si è avuto modo di vedere, è definita nel secondo libro della Fisica mettendo in gioco essenzialmente due significati: in un senso essa è forma e in un altro materia. ${ }^{34}$

La movenza che consiste nell'alternanza di punti di vista si fa evidentissima nella trattazione aristotelica del tempo. Infatti, lo Stagirita mette in campo due accezioni di tempo:

$\overline{{ }^{34} \text { Cfr. Fisica, B, 1, } 193}$ a.

Educ. e Filos. Uberlândia, v. 23, n 45, p. 101-164, jan./jun. 2009. 
1. il tempo inteso come infinito; cioè diremmo la dimensione del tempo "in sé";

2. Il tempo assunto di volta in volta, dunque, il tempo "per noi" che, a differenza del primo, è da intendersi come finito, o meglio, che noi percepiamo di volta in volta come tale.

Ancora, tempo e movimento sono legati con un rapporto biunivoco, ma anche nel definire tale rapporto è possibile una duplice considerazione a partire dalla duplice funzione che Aristotele attribuisce al numero.

1. Infatti, il tempo può dirsi "numero del movimento", cioè numerato dal movimento, stando al primo senso in cui

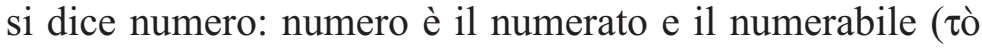

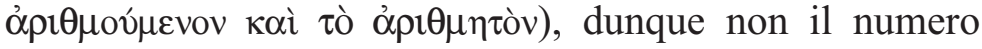
come unità di misura, ma l'oggetto reale numerato. Inteso in questo senso, il tempo risulta, quindi, sempre diverso nel suo scorrere (Fisica, $\Delta, 11,219$ b);

2. ora, però, se il numero viene considerato nel suo secondo senso, come il mezzo attraverso cui numeriamo $(\hat{\omega}$

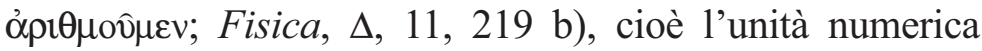
corrispondente al numero, esso è sempre identico (anche se applicato ad oggetti diversi) $)^{35}$. Di conseguenza, in questa

\footnotetext{
${ }^{35}$ Wieland (Fisica di Aristotele..., p. 402, p. 404, p. 407) precisa che quello aristotelico "non è un concetto astratto di unità, ma ha sempre anche una definizione di contenuto. Essa si regola secondo il corrispondente ambito concettuale"; egli fonda questa sua posizione facendo riferimento a Metafisica N, 1,1087 b 34, ma questa stessa concezione dell'unità di misura si rintraccia anche nella Fisica proprio nel contesto di questa stessa trattazione del tempo. Infatti, Aristotele - definendo la conversione circolare come unità di misura del tempo - afferma che "ciascuna cosa si misura con qualcosa di affine (le monadi con le monadi, i cavalli con i cavalli)" (223 b 15), cosa che conferma ulteriormente la lettura di Wieland. In questo senso non è del tutto esatto quanto afferma Callahan, (J.F. Callahan, Four views of time in ancient philosophy, Harvard University
} 
seconda accezione, il tempo si dice numero come misura del movimento e risulta sempre identico (Fisica, $\Delta, 12,220 \mathrm{~b}$ ).

Infine, Aristotele riconosce una duplice funzionalità anche all'istante rispetto al tempo:

1. l'istante rende continuo il tempo, perché è quell'estremità in comune tra prima e poi e Aristotele definisce continuo ciò che ha un'estremità in comune (Fisica, Z, 1, $231 \mathrm{a}$ );

2. l'istante divide, perché è un limite inteso come principio del futuro e fine del passato.

E' evidente, dunque, come la volontà aristotelica di comprendere e spiegare la realtà in tutta la sua complessità imponga ad Aristotele un approccio gnoseologico ricco di sfumature e continue distinzioni e, dunque, esso stesso profondamente complesso.

\section{La ricchezza gnoseologica come chiave per spiegare la radicale pluralità metodologica}

Una tale ricchezza gnoseologica sembra avere una ricaduta immediata a livello metodologico. L'atteggiamento aristotelico nei confronti del metodo è, infatti, estremamente duttile e disinvolto, come è evidente dal brano del De anima analizzato sopra (A, 1, 403 a-b): lo Stagirita qui afferma che fisico e dialettico definiscono lo stesso oggetto, la collera o la casa, in modo estremamente diverso. "Vediamo, così, ben esemplificata in poche righe la possibilità di avere, intorno ad uno stesso oggetto, approcci legittimi e anche molto diversi. Tuttavia, se ci fermassimo a questo livello di riflessione, sarebbe possibile sostenere che la molteplicità metodica sia connessa

Press, Cambridge, 1948, p. 50) che, commentando questa distinzione parla, nel primo senso, di numero inteso come oggetto reale numerato e, nel secondo senso, di numero come concetto astratto. Wieland precisa, infatti, che il numero in quanto numero (1052 b 24), cioè appunto, la mera astrazione viene inteso da Aristotele come un "concetto predicativo" che, in quanto tale - e in polemica con la concezione platonica delle Idee-Numero - non è affatto autonomo. 
esclusivamente alla distinzione di ambiti; si tratterebbe di un dato reale, ma relativamente importante. La posizione di Aristotele sul metodo è molto più radicale". ${ }^{36}$

Ancora nel De anima, egli riconosce che

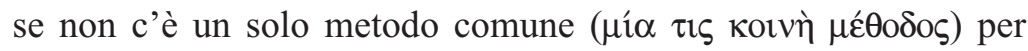
determinare che cos'è una cosa, la trattazione diventa ancora più difficile, infatti bisognerà stabilire un certo modo di procedere per ciascun oggetto (A, 1, 402 a 16-19).

La diversità degli oggetti e degli ambiti di ricerca, dunque, è sicuramente un dato che in parte spiega i diversi approcci messi in atto da Aristotele, ma non è sufficiente per giustificare la radicalità di un tale atteggiamento, testimoniato in modo paradigmatico da un passo del De generatione et corruptione, in cui egli critica il modo troppo semplicistico nel quale Empedocle ha affrontato il problema del movimento:

bisognava, pertanto, o definire o porre delle ipotesi o fare delle dimostrazioni, svolte $\mathrm{o}$ con rigore $\mathrm{o}$ in modo più debole $\mathrm{o}$ in qualche altro modo. (De generatione et corruptione $\mathrm{B}, 6,333$ b 24-26).

Si vede bene, dunque, come lo Stagirita inviti ad usare una pluralità di strumenti anche per affrontare un singolo e specifico problema. ${ }^{37}$

Alla luce della movenza gnoseologica appena illustrata, sembra plausibile pensare, dunque, che la radicale varietà di approcci metodologici che Aristotele adotta - e che, per quanto riguarda in particolare le opere fisiche, trova una ragione immediata

${ }^{36}$ Migliori, Divino e umano. L'anima in Aristotele e in Platone, in AA. VV., Mente, anima e corpo nel mondo antico, a cura di U. la Palombara e G. A. Lucchetta, Opera Editrice, Pescara, 2006, pp. 21-56, p. 22.

${ }^{37}$ Cfr. Migliori, Aristotele, Generazione..., p. 18, n. 13. 
nella estrema varietà e vastità degli oggetti della scienza fisica - sia l'espressione, a livello metodologico, di questo suo atteggiamento epistemico di fondo che consiste nel mettere in gioco molteplici schemi interpretativi per spiegare una medesima realtà.

Nel contesto di alcune opere fisiche, infatti, la complessità delle diverse prospettive di analisi del reale pare assumere un'articolazione più propriamente metodologica, perché Aristotele utilizza costantemente nell'argomentazione due punti di vista che indica con due avverbi: $\varphi v \sigma ı \omega \hat{\varsigma}$ e $\kappa \alpha \theta \hat{\lambda} \lambda$ ov, cioè egli affronta un dato argomento inerente al mondo della natura (o lo confuta, nel caso ci si trovi nella sezione dedicata agli endoxa dei predecessori) da una parte con argomenti inerenti alla scienza della natura ${ }^{38}$ e dall'altra con argomenti di valore più generale, svolgendo, ovviamente, trattazioni parallele e diverse fra di loro.

Un buon esempio di questo procedimento è fornito dal primo libro del De caelo, dove molte argomentazioni (quali la finitezza e l'unicità del kosmos $)^{39}$ vengono sostenute mettendo costantemente in campo questo duplice punto di vista.

Un'analoga distinzione è riscontrabile anche in Fisica, $\Gamma$ nel contesto della trattazione dell'infinito. Infatti, per dimostrare che non esiste un corpo infinito, Aristotele procede innanzitutto ricorrendo

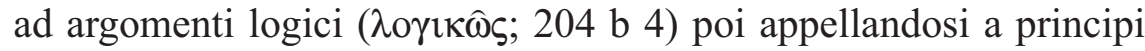
fisici ( $\varphi v \sigma \iota \kappa \hat{\omega} \varsigma ; 204$ b 10).

Ancora nel De caelo, risulta particolarmente significativa l'applicazione di questa duplice considerazione nel contesto critico

\footnotetext{
${ }^{38}$ Mansion (Introduction..., p. 222) nota che tale metodo esplicativo "dato che è in contatto con la realtà osservata, è veramente appropriato alla filosofia della natura". Egli osserva poi però che le considerazioni di carattere fisico "si oppongono a quelle che sono puramente dialettiche" che non costituiscono il metodo proprio della scienza fisica. A me pare evidente, invece, che non ci sia affatto un rapporto di opposizione, ma semplicemente l'applicazione di due punti di vista diversi allo stesso oggetto.

${ }^{39}$ De caelo, A, 5, 271 b; A, 7, 276 a.
} 
riguardante il problema dell'eternità del kosmos (A, 10, 279 b). Infatti, rispetto alla teoria platonica relativa a questo problema, lo Stagirita conduce una critica che si articola esattamente in due sezioni indicate dallo stesso Aristotele: una sezione propone argomenti critici

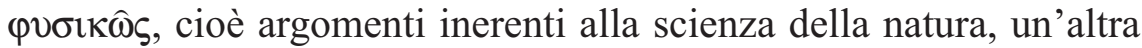
$\kappa \alpha \theta \hat{\lambda}$ ov, cioè argomenti di valenza generale, in questo caso logica:

abbiamo mosso loro obiezioni con argomenti propri della

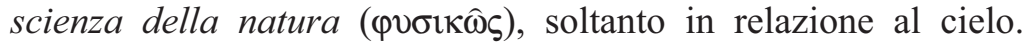
Ma conducendo un esame in generale ( $\kappa \alpha \theta \dot{\lambda} \lambda \mathrm{ov})$ rispetto ad ogni realtà, sarà chiara anche tale questione (280 a 32-34).

Dunque, analogamente ai diversi punti di vista con i quali guarda alla realtà, lo Stagirita usa diversi schemi metodologici che corrispondono alle diverse finalità che, di volta in volta, egli si pone e ai diversi punti di osservazione e di partenza attraverso cui si avvicina all'analisi di una realtà.

\section{I diversi metodi applicabili alla scienza della natura}

A riprova di quanto fin qui argomentato, nell'opera fisica è possibile rintracciare movenze metodologiche diverse, che rispondono a fini diversi e che danno vita a un quadro composito e variegato che permette di riconoscere la compresenza di più metodi applicabili per avere scienza della natura.

5.1 Un approccio dialettico: l'analisi degli endoxa dei predecessori

Una movenza costante nelle opere fisiche e biologiche è quella di affrontare un dato problema a partire dall'analisi delle opinioni in proposito dei predecessori, con la finalità di riconoscere gli eventuali errori, di rilevare le aporie e di superarle formulando una nuova teoria. 
Questo schema metodologico viene teorizzato in modo particolarmente chiaro nel De caelo rispetto al problema della generazione o dell'eternità del kosmos:

1. si pone il problema: "diciamo se (il mondo) è ingenerato o generato o se è incorruttibile o corruttibile" (De caelo, 279 b 4-5);

2. per prima cosa si passano in rassegna "le opinioni degli

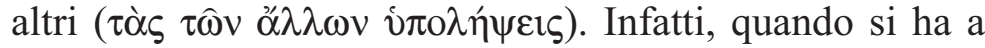
che fare con tesi contrarie, le dimostrazioni delle une costituiscono altrettante aporie per le altre" (De caelo, 279 b 5-12); inoltre questo passaggio permette di comportarsi come arbitri e di non condannare in contumacia. Si tratta, dunque, di un momento funzionale a capire che cosa $\mathrm{i}$ filosofi precedenti hanno detto di valido e a criticare - e quindi eliminare - gli errori. ${ }^{40}$

3. Dalla critica delle posizioni opposte emerge, e rimane stabilita, la posizione aristotelica che supera le aporie rilevate nelle soluzioni dei predecessori. ${ }^{41}$

Dal quadro appena ricostruito, risulta evidente come Aristotele usi gli $\tilde{\varepsilon} v \delta o \xi \alpha$ : egli li intende come un terreno sul quale operare dialetticamente. ${ }^{42}$ Infatti, "se si riesce a mostrare non solo che una

${ }^{40}$ Vegetti (Aristotele, Opere biologiche, a cura di D. Lanza e M. Vegetti, U.T.E.T., Torino, 1971, p. 26) nota, inoltre, che "ad Aristotele importa, una volta individuato un problema, scoprire se nelle ricerche che lo hanno preceduto sia in qualche modo riscontrabile un presentimento e conseguentemente una risposta per quanto implicita alla questione che egli pone".

${ }^{41}$ Questo procedimento, esposto in modo così esplicito, si rintraccia almeno un'altra volta nel De caelo in relazione alla questione del peso e della leggerezza: "pertanto, dopo aver esaminato, innanzitutto, ciò che sostengono gli altri e dopo aver rilevato le aporie che è necessario risolvere in relazione a questa ricerca, diremo anche ciò che noi pensiamo a questo proposito" $(\Delta, 1,308$ a 4-7).

${ }^{42}$ Appare, dunque, troppo netta la posizione di Bolton (R. Bolton, Aristotle's 
tesi lascia sussistere questi $\check{\varepsilon} v \delta o \xi \alpha$, ma anche che la tesi opposta, la quale costituisce per essa una difficoltà, può essere confutata, e quindi questa difficoltà può essere risolta, a partire dallo stesso insieme di $\varepsilon^{\prime} v \delta o \xi \alpha$, allora - conclude Aristotele - si sarà dimostrato in modo sufficiente (la prima tesi). ${ }^{43}$

La finalità di un tale approccio, dunque, è critico-costruttiva: criticando ed eliminando gli errori si riesce a stabilire una teoria che supera le aporie rilevate. ${ }^{44}$

Tale finalità è ribadita in modo altrettanto chiaro nel quarto libro della Fisica, in cui lo Stagirita, trattando del luogo, descrive un modo di procedere propriamente dialettico, nel quale si giunge alla soluzione attraverso la trattazione delle aporie rilevate tramite la discussione delle posizioni dei predecessori.

Bisogna tentare di condurre la ricerca in modo tale da definire che cos'è questo oggetto, da sciogliere le aporie e che quelle proprietà che sembrano appartenere al luogo, gli appartengano effettivamente; e inoltre, che sia chiara la causa della difficoltà e delle aporie inerenti a questo oggetto: così, infatti, ogni cosa potrà essere dimostrata nel modo migliore $(\Delta, 4,211$ a 7-11).

\footnotetext{
Method in Natural Science, in Aristotle's Physics: a collection of Essays, Edited by L. Judson, Clarendon Press, Oxford, 1991, pp. 1-29, p. 11), il quale sostiene che il metodo della scienza naturale non può dirsi dialettico, perché la dialettica prende avvio direttamente dalla discussione degli endoxa, mentre la fisica dall'esperienza, cui possono aggiungersi gli endoxa, dei quali, però, non ha bisogno. Ora, nell'argomentazione fisica aristotelica avremo modo di verificare l'importanza dell'esperienza, ma, come testimonia il passo del De caelo appena citato e quelli che seguiranno, anche il procedimento dialettico non solo trova sicuramente spazio ma, al contrario di quanto afferma Bolton, anche in campo fisico sembra poter dare avvio alla ricerca.

${ }^{43}$ E. Berti, Nuovi studi aristotelici. Epistemologia, logica e dialettica, volume I, Morcelliana, Brescia, 2004, p. 275.

${ }^{44}$ In questo senso, "quel che lo (Aristotele) spinge a confrontarsi con i contributi precedenti è la convinzione di un progresso nelle conoscenze e nelle tecniche» (Migliori, Aristotele, Generazione..., p. 39).
}

Educ. e Filos. Uberlândia, v. 23, n 45, p. 101-164, jan./jun. 2009. 
Questo passo fa rilevare un'ulteriore finalità riconoscibile nel procedimento dialettico: esso permette anche di capire le ragioni che hanno condotto i predecessori a fare determinate ipotesi e quali sono i problemi teorici che un dato oggetto comporta. In linea con questo approccio, Aristotele dedica l'ultima sezione del primo libro (A, 8-9) - nella quale si stabiliscono i principi naturali di forma, materia (o sostrato) e privazione - alla discussione delle opinioni dei suoi predecessori perché

solo così si scioglie anche la difficoltà degli antichi. Infatti, quelli che per primi fecero filosofia ricercando la verità e la natura degli esseri, furono, per così dire, sviati, spinti dall'inesperienza (A, 8, 191 a $23-27)^{45}$.

Questo stesso modo di procedere si rintraccia nel De anima, dove, subito dopo aver inquadrato il tipo di ricerca, Aristotele la imposta secondo questi tre passaggi.

Nel condurre una ricerca intorno all'anima è necessario, insieme all'esame sulle difficoltà che si devono risolvere procedendo, raccogliere inoltre le opinioni dei predecessori $(\tau \hat{\omega} \nu \pi \rho \tau \tau \dot{\varepsilon} \rho \omega \nu$ $\delta$ ó $\xi \alpha \varsigma$ ), quanti hanno detto qualcosa di essa, per accogliere ciò che hanno detto bene e stare attenti a ciò che potrebbe non essere corretto (403 b 20-25).

\footnotetext{
${ }^{45}$ Passi confrontabili all'interno della Fisica si rintracciano rispetto al movimento e alla trattazione sul primo motore: rispettivamente $\Gamma, 2,201 \mathrm{~b}$ e libri $\mathrm{H}$ e $\Theta$; rispetto all'infinito: $\Gamma, 4,203$ a; rispetto al vuoto dove Aristotele afferma chiaramente che "bisogna, comunque, iniziare la ricerca prendendo in esame la teorie di quelli che ne sostengono l'esistenza e di quelli che, al contrario, la negano, e in terzo luogo le opinioni comuni su tali argomenti" $(\Delta, 6,20-$ 22). Un confronto continuo, anche se non sempre così sistematico ed esplicito, con i predecessori è comunque rintracciabile anche nella trattazione del luogo (soprattutto $\Delta, 2)$ e del tempo $(\Delta, 10,218 \mathrm{~b})$.
} 
Non sfugge a questa procedura metodologica neanche il De generatione et corruptione in cui quasi ogni trattazione si apre presentando la questione che si discuterà e poi passando in rassegna le opinioni di chi, tra i predecessori, ha trattato quello stesso argomento. Per esempio, l'incipit stesso del trattato presenta un elenco delle varie e diverse opinioni dei predecessori in merito alla generazione e all'alterazione (A, 1, 314 a 1-8) e, dalla loro confutazione, si chiarisce via via il pensiero aristotelico. ${ }^{46}$

A ulteriore dimostrazione della peculiarità di tale modo di procedere, esso è rintracciabile anche in un trattato "minore" come il De respiratione, trattazione che si colloca all'interno dei Parva Naturalia.

Infatti, nel trattato sulla giovinezza e la vecchiaia, la vita e la morte, si pone un excursus sulla respirazione che si apre proprio con l'analisi delle posizioni dei predecessori, analogamente a quanto accade nei trattati "maggiori".

Pochi fisici precedenti hanno parlato della respirazione. Tuttavia per quale fine appartenga agli animali, alcuni non lo hanno detto, altri lo hanno detto, però non ne hanno parlato correttamente, ma

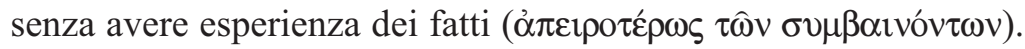
Inoltre, affermano che tutti gli animali respirano, il che non è vero. Quindi, è necessario esaminare innanzitutto queste cose, perché non sembri che giudichiamo in modo vano gli assenti (470 b 6-12).

\footnotetext{
${ }^{46}$ Lo stesso procedimento è attuato in $\mathrm{A}, 2,315 \mathrm{a}-\mathrm{b}$, a proposito della generazione $\mathrm{e}$ corruzione assolute; in A, 7, 323 b e A, 8, 324 b-325 a, dove si discute dell'azione e della passione; in A, 10, 327 a, in cui Aristotele tratta della mescolanza; in B, 1, 328 b-329 a, dove Aristotele discute degli elementi e della materia prima; in B, 6, 333 a dove Aristotele svolge una complessa confutazione della posizione di Empedocle; in B, 7, 333 b, in cui la trattazione riguarda la formazione dei corpi; in $\mathrm{B}, 9,335 \mathrm{~b}$ dove Aristotele tratta della causa efficiente.
} 
Ritornano, dunque, $\mathrm{i}$ tre passaggi di cui si è parlato e Aristotele li giustifica allo stesso modo:

1. si pone il problema (che, in questo caso, è da porre alle linee 470 b 4-5 dove appunto si dice che gli animali si raffreddano con aria e acqua, ma bisogna approfondire in quale modo);

2. si espongono le opinioni dei predecessori, confutate poi attraverso le proprie teorie, affinché non sembri che si giudichi vanamente;

3. si propone la propria soluzione.

Una variazione rispetto a questa procedura è rilevabile, invece, proprio negli altri trattati che costituiscono i Parva Naturalia. Lo Stagirita sembra, infatti, prediligere qui una modalità più schematica. L'incipit di ogni piccolo trattato propone un elenco di problemi, ai quali, quasi sempre in modo ordinato, Aristotele dà risposta, sottolineando che il problema posto è stato risolto (anche se la soluzione aristotelica sembra esposta in maniera molto stringata: lo Stagirita si attiene sempre scrupolosamente alla tematica in oggetto, divagando poco e, quando lo fa, limitandosi ad accennare a questioni già affrontate o che vengono rimandate ad altre argomentazioni all'interno dei Parva per una trattazione più completa $\left.{ }^{47}\right)$. Un bell'esempio in questo senso è offerto dall'incipit del trattato De somno:

Circa il sonno e la veglia bisogna esaminare $<1>$ che cosa potrebbero essere e $<2>$ se siano propri dell'anima o del corpo, oppure comuni e se fossero comuni, $<3>$ di quale parte dell'anima o del corpo e $<4>$ per quale causa appartengono agli animali e $<5>$ se tutti partecipano

\footnotetext{
${ }^{47} \mathrm{Cfr}$. ad esempio, De insomniis, 1, 458 b 1-10; De longitudine et brevitate vitae, 1,464 b 30-465 a 2.
} 
di entrambi o alcuni di uno e alcuni dell'altro soltanto o alcuni di nessuno e altri di entrambi. Oltre a queste cose, $<6>$ che cosa è il sogno e $<7>$ per quale ragione chi dorme talvolta sogna talvolta non sogna $\mathrm{o}<8>$ se a chi dorme accade sempre di sognare, ma non lo ricorda e se questo accade, per quale ragione avviene. $<9>\mathrm{E}$ se sia possibile o non sia possibile prevedere gli eventi che verranno e $<10>$ in quale modo se è possibile; se gli eventi futuri che dipendono dall'uomo soltanto o anche tra quelli che hanno come causa il divino o si generano per natura o per caso (453 b 11-24).

Rispetto alla metodologia riscontrata in trattati di più ampia estensione, dei tre passaggi rilevati sembra manchi quasi del tutto il secondo, o meglio, la critica ai predecessori è condotta in modo molto meno sistematico (nella maggior parte dei casi non ha un "luogo" ad essa riservato, ma si ricava da note e passaggi veloci) ed è molto più frettolosa.

In questo modo di procedere, inoltre, si rileva una costante inversione del secondo e del terzo punto: mentre negli altri trattati la critica ai predecessori solitamente precede la risposta aristotelica, qui - pur sempre in modo non sistematico - sembra avvenga il contrario. ${ }^{48}$

\footnotetext{
${ }^{48}$ Indicativa è la trattazione della vista nel De sensu, 2, A 437 a-b. Aristotele prende in considerazione le teorie di Empedocle, Platone e Democrito in proposito, intrecciando il loro punto di vista alle sue considerazioni e dando così luogo a velocissime, e talvolta, dal punto di vista storico, inesatte, testimonianze e confutazioni; lo stesso accade nella trattazione dei sapori in cui vengono prese di mira le opinioni di Empedocle e Democrito (De sensu, 4, 440 b - 441 a); ancora più evidente in De sensu, $6,445 \mathrm{~b}$, dove la critica a Empedole è sottesa a tutta l'argomentazione, ma non trova un luogo proprio; infine, nel De iuventute si riscontrata un puntuale riferimento al Timeo platonico che però - oltre ad essere raramente esplicito - non si colloca in una sezione espressamente dedicata ai predecessori, ma appunto si confonde nel corso della trattazione aristotelica. Una riflessione dedicata ai predecessori risulta, invece, del tutto assente nel De memoria, nel De somno, nel De insomniis, nel De divinatione, nel De longitudine et brevitate vitae.
} 
Tale inversione fa sì che questo momento di critica tenda a non avere più né la funzione di rilevare e salvare il giusto nelle dottrine dei predecessori né quella di criticare gli errori come farebbe un arbitro.

In tal modo, si crea - con maggiore evidenza che in altri trattati - la dinamica per cui Aristotele, sulla base della propria posizione, critica le dottrine altrui, correndo il rischio effettivo di applicare ai predecessori categorie e distinzioni che essi non potevano conoscere e/o utilizzare. Tale rischio, comunque, è sempre presente nella critica aristotelica che ha uno spiccato valore teoretico e non storico. ${ }^{49}$

Del resto, l'assenza della sezione dedicata agli endoxa dei predecessori si registra anche in altri trattati biologici dal carattere molto tecnico, quali l'Historia animalium o il De incessu animalium, il che fa ipotizzare che in trattati specifici - nei quali si riduce il contributo teorico, mentre preponderante è la fenomenologia - ci sia meno bisogno di un costante confronto dialettico. Certo, un tale procedimento potrebbe anche essere giustificato, a seconda dei casi, dal fatto che su alcuni di questi argomenti i predecessori fornivano poco materiale o dal fatto che si tratta di lezioni rapide, in cui non c'è un grande interesse filosofico.

Oltre che a semplici tesi, questa stessa operazione dialettica può essere applicata in relazione ai principi stessi di una scienza $\mathrm{e}$, in questo contesto, Aristotele sembra riconoscere all'approccio dialettico la finalità di condurre alla scoperta dei principi delle scienze, perché un tale procedimento "permette di discernere, tra le diverse opinioni che sono, per così dire, i candidati al titolo di principi di

\footnotetext{
49 "Aristotele insegnò a fare la storia dei problemi premettendo a molte delle sue trattazioni una rassegna delle opinioni dei precursori: dove tuttavia parlare di "storia" è particolarmente improprio, poiché le rassegne hanno fondamentalmente il compito di fornire alla riflessione del filosofo quel materiale di fatti, o di discorsi sui fatti, che saranno poi sottoposti al vaglio della critica e alla prova del collaudato armamentario dei concetti aristotelici" (P. Donini, La filosofia di Aristotele, Loescher, Torino, 1982, p. 24).
} 
una scienza, quali sono vere, e dunque sono dei veri principi, e quali non lo sono". ${ }^{50}$ Il procedimento resta, dunque esattamente lo stesso, ma a cambiare è il campo di applicazione: se nei casi finora visti tale metodo si applica a semplici tesi, in questo caso, in discussione sono i principi stessi e, vagliando i vari દ̌v $\delta \circ \xi \alpha$, si giunge a stabilire la verità dei principi. ${ }^{51}$

Un buon esempio di questa applicazione sul terreno protologico del procedimento dialettico è costituito proprio dal primo libro della Fisica, nel quale Aristotele discute, confrontandosi con i predecessori, la questione dei principi fondamentali dell'essere per introdurre la scienza della natura, pur riconoscendo l'estraneità di tali argomenti rispetto alla fisica strictu senso. ${ }^{52}$

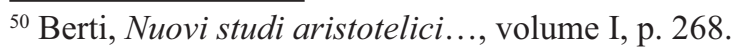

${ }^{51}$ Berti (Nuovi studi aristotelici..., volume I, p. 268) osserva che "la sola differenza che rimane [...] tra l'impiego della dialettica a proposito di tesi che non sono principi e il suo impiego a proposito dei principi, è che nel primo caso la dialettica non è indispensabile, mentre nel secondo sembra esserlo", anche se lo stesso studioso riconosce che tale questione è comunque problematica, perché un altro procedimento di scoperta dei principi è quello induttivo; egli conclude che "resta stabilito che la dialettica permette di scoprire i principi per mezzo del suo

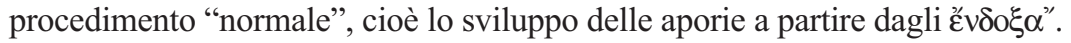

${ }^{52}$ Berti (E. Berti, Les méthodes d'argumentation et de démonstration dans la "Physique" (apories, phénomènes, principes), in Physique..., pp. 51-72, p. 59) sottolinea, infatti, che, la confutazione degli Eleati nel primo libro della Fisica pone di fronte ad un "programma di ricerca che non coincide con la fisica, ma la precede come una sorta di fondazione della fisica o meglio di ricerca delle sue condizioni di possibilità [...]. Ma fino a questo punto non si è ancora nella fisica; si è semplicemente di fronte a una specie di introduzione di carattere dialetticometafisico". Così anche Ross (W.D. Ross, Aristotle's Physics, a revised text with introduction and commentary by W.D. Ross, Clarendon Press, Oxford, 1936, $1955^{2}$, pp. 19-20), il quale afferma che "il primo libro della Fisica annuncia che il suo oggetto è la natura, ma non tenta di definire che cosa sia la natura. Invece di fare questo, il suo obiettivo è quello di determinare una volta per tutte i primi principi, le necessarie condizioni e gli elementi della natura”. Infatti, a riprova della natura introduttiva del primo libro rispetto alla trattazione propriamente fisica, Aristotele, chiudendolo, rimanda la trattazione dei principi metafisici $\mathrm{ad}$ un contesto più adeguato e anticipa gli argomenti che seguiranno, tenendo 
Dal momento che in ogni ricerca, di cui ci siano principi o cause o elementi, il sapere e la scienza dipendono da questi - infatti crediamo di conoscere ciascuna cosa, quando conosciamo le cause

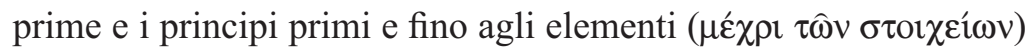
- è evidente che anche riguardo alla scienza della natura si deve tentare di determinare prima di tutto ciò che riguarda i principi

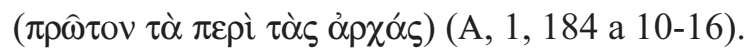

La movenza discensiva - dai principi agli elementi - qui descritta da Aristotele, è funzionale ad un procedimento scientifico dimostrativo, secondo il quale si ha scienza di un oggetto quando dai suoi principi si è capaci di discendere fino agli elementi. In questo senso, il punto di partenza di un tale procedimento sono i principi. Infatti, la discussione con i predecessori affronta la tematica dell'Essere e dei principi metafisici (A, 2-6), "ma, dal momento che, anche se non trattano di fisica, a loro capita di parlare di difficoltà legate alla fisica, è forse bene discuterne un po': infatti, in questa ricerca c'è filosofia" (A, 2, 185 a 18-20).

Il valore di verità degli endoxa

Se, dunque, come si è appena verificato, il procedimento dialettico operato da Aristotele si basa sugli $\tilde{\varepsilon} v \delta o \xi \alpha$, per misurare il "grado di verità scientifica" di tale metodo, si deve stabilire il valore epistemologico che lo Stagirita attribuisce agli ع̌vঠo $\xi \alpha$.

Ora, la grande fiducia di Aristotele nella razionalità umana

presente però la base argomentativa e metodologica data qui: "Determinare poi il principio formale, se ce ne sia uno o ce ne siano molti e quale esso sia o quali siano, è compito della filosofia prima e perciò la ricerca si rinvii a quella sede. Nei prossimi trattati discuteremo invece delle forme fisiche e corruttibili. Pertanto, che vi siano dei principi e quali e quanti per numero, rimanga da noi stabilito nel modo che abbiamo seguito finora. D'ora in poi, invece, si continui, muovendo da un altro punto di vista" (A, 9, 192 a 35- 192 b 5). 
lo porta a pensare che ciò che è condiviso dai più debba avere un certo valore di verità ${ }^{53}$, precisando il fatto che la condivisione indica la coerenza degli endoxa tra di loro: "ciò che rende degno

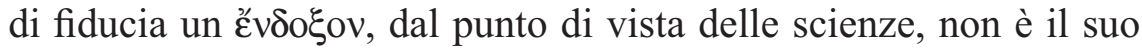
grado di endossalità, cioè la quantità o la qualità delle persone che lo

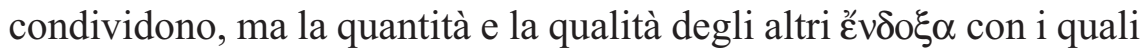
esso è o non è compatibile". ${ }^{54} \mathrm{Si}$ tratta, dunque, di un atteggiamento "paradigmatico".

La natura degli ع̌v $\delta \circ \xi \alpha$ è espressa con chiarezza in un passo dei Topici dove lo Stagirita li definisce come un particolare tipo di opinioni, ${ }^{55}$ " $<1>$ di tutti $\mathrm{o}<2>$ dei più $\mathrm{o}<3>$ dei sapienti e, tra questi, $\mathrm{o}<3.1>$ di tutti, $\mathrm{o}<3.2>$ dei più, $\mathrm{o}<3.3>$ dei più noti e illustri" (Topici, A, 2, 100 b 21-23).

Le diverse sfaccettature evidenti nella definizione stessa aprono lo spazio anche a contrasti tra opinione comune e opinione filosofica e quindi alla necessità di confrontare a fondo le varie tesi.

Il valore epistemologico degli ع́v $\delta \circ \xi \alpha$ viene puntualizzato in un passaggio della Retorica, nel quale Aristotele afferma:

\footnotetext{
53 "Secondo Aristotele è probabile che l'opinione corrente contenga una parte di verità, come è probabile che le opinioni dei saggi non siano mai del tutto sbagliate. "Chiunque può apportare un contributo". Un attento esame delle "opinioni", dunque, non solo porterà alla luce i problemi, ma metterà anche a disposizione gran parte del materiale atto a risolverli" (J.L., Ackrill, Aristotle the Philosopher, Oxford University Press, Oxford, New York, Toronto, 1981; traduzione italiana di P. Crivelli, Aristotele, Il Mulino, Bologna, 1993, p. 178). Berti (Nuovi studi aristotelici..., volume I, p. 273), su questa linea, osserva che "per Aristotele la coerenza di una proposizione con la maggior parte degli

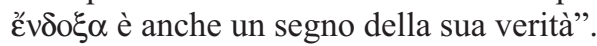

${ }^{54}$ Berti, Nuovi studi aristotelici..., volume I, p. 273.

${ }^{55}$ Berti (Nuovi studi aristotelici..., volume I, p. 279), commentando il passo di Retorica, B, 25, 1402 a 32-34 - nel quale, secondo alcune interpretazioni, Aristotele sosterrebbe che ci siano $\varepsilon^{\prime} v \delta \circ \xi \alpha$ falsi, ricorda che "le opinioni ( $\tau \grave{\alpha}$

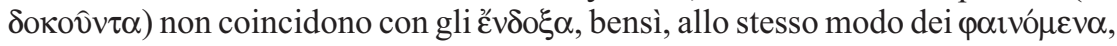
li comprendono".
} 
[...] infatti, spetta alla stessa facoltà conoscere il vero e ciò che è

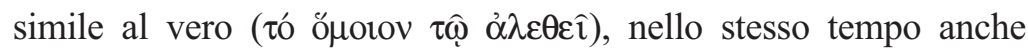
gli uomini sono adeguatamente disposti per natura verso il vero e nella maggior parte dei casi colgono la verità. Perciò, l'essere ben

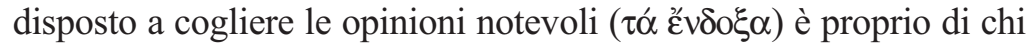
è ugualmente disposto verso la verità (Retorica, A, 1, 1355 a 1a-18).

Da qui si ricava, dunque, che gli ह̌v $\delta \circ \xi \alpha$ sono vicini alla verità, sono "ciò che è simile al vero", "nel senso migliore del termine "simile", quello per cui esso significa "quasi uguale", cioè "prossimo al vero". ${ }^{56}$

Aristotele introduce, poi, nel secondo capitolo della Retorica, il concetto di "vero interamente o nella maggior parte dei casi ( $讠$

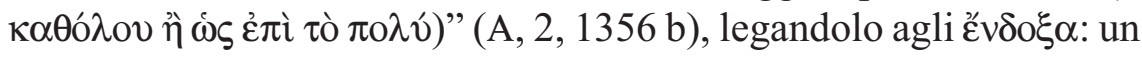

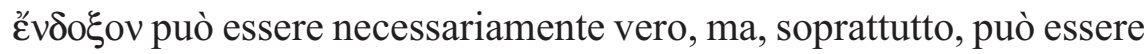
vero nella maggior parte dei casi. Ora, in questa seconda accezione,

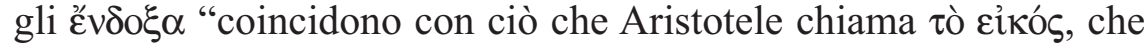
significa ciò che è simile al vero $[\ldots]$ non nel senso che non sia vero, ma nel senso che è vero nella maggior parte dei casi', ${ }^{57} \mathrm{E}$ ' evidente che, tale valenza epistemologica è del tutto spendibile in una scienza come la fisica che è la scienza delle cose che accadono "sempre o

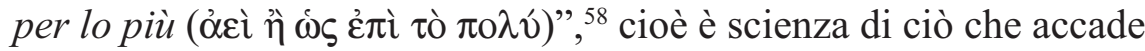
nella maggior parte dei casi. Dunque, "la base epistemologica della dialettica aristotelica è costituita dal fatto che gli ع́v $\delta \circ \xi \alpha$ nella loro

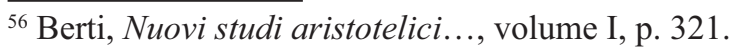

${ }^{57}$ Berti, Nuovi studi aristotelici..., volume I, p. 323.

${ }^{58}$ Cfr., Fisica, B, 8, 198 b 35-36. Questa concezione trova conferma anche nelle opere biologiche, citiamo a titolo di esempio un passo del De partibus animalium: "Bisogna studiare la natura, osservando la maggioranza dei casi;

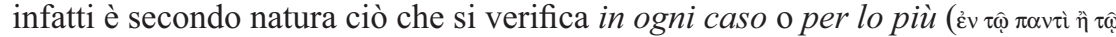

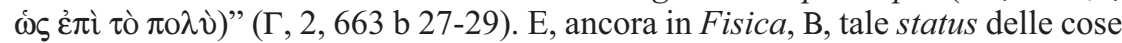
naturali è distinto da ciò che accade per caso: "infatti, queste cose e tutto ciò che

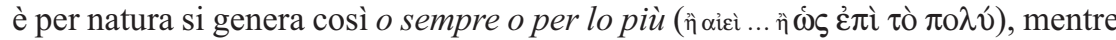
non è così per le cose fortuite o casuali" (B, 8, 198 b 35).
} 
maggior parte sono anche veri e per questa ragione permettono di vedere, tra due tesi opposte da cui si siano dedotte le conseguenze, quale è vera e quale è falsa, l'una essendo quella le cui conseguenze si accordano con la maggior parte degli हैv $\delta \circ \xi \alpha$, l'altra quella le cui conseguenze vi contraddicono". 59

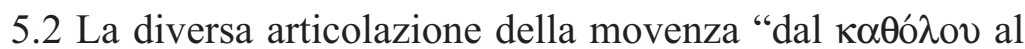
$\kappa \alpha \theta$ 'ย̌ $\alpha \sigma \tau \alpha$ "

Una movenza diversa da quella dialettica è quella che Aristotele descrive con l'espressione "procedere dal generale al particolare". In questo caso, infatti, nel contesto dell'opera fisica, il punto di partenza non è costituito dagli endoxa dei predecessori, ma da ciò che si presenta come primo o più evidente alla sensazione.

La precisazione del terreno nel quale ci troviamo è però d'obbligo, perché tale approccio ci pone, innanzitutto di fronte a un problema terminologico e al rischio di un equivoco: infatti, come vedremo, in opere e in contesti diversi lo Stagirita utilizza sempre

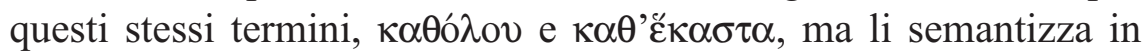
modo diverso. Per questo, un'espressione che generalmente, indica un processo deduttivo (intendendo $\kappa \alpha \theta$ ó $\lambda \mathrm{ov}$ come "universale" e $\kappa \alpha \theta$ 'ย̌ $\alpha \sigma \tau \alpha$ come "particolare"), nel contesto fisico, viene ad indicare un processo che potremmo definire induttivo, ma che si articola in modi diversi nelle diverse opere (per esempio, si può

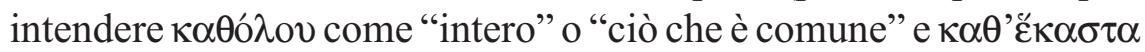
come "principio" o "elemento costitutivo"). Per evitare l'equivoco bisogna, dunque, ricostruire con attenzione i diversi contesti e le diverse finalità che lo Stagirita si pone con la sua analisi.

\footnotetext{
${ }^{59}$ Berti, Nuovi studi aristotelici..., volume I, p. 280; il quale riconosce anche che

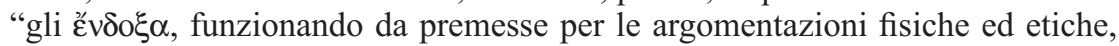
ci permettono di ottenere conclusioni che hanno valore scientifico, nello stesso senso in cui possono essere considerate scienze filosofiche la fisica e l'etica" (p. $330)$.
}

Educ. e Filos. Uberlândia, v. 23, n 45, p. 101-164, jan./jun. 2009. 
A. Da ciò che è più chiaro per noi a ciò che è più chiaro per natura: la Fisica

Nell'incipit della Fisica, Aristotele sembra descrivere un processo epistemico che definiremmo induttivo:

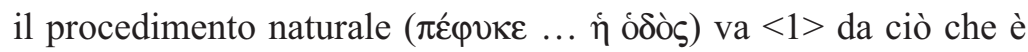

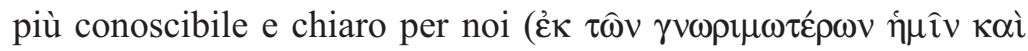

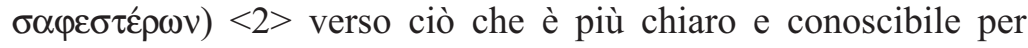

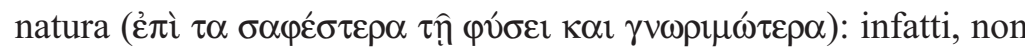
è lo stesso il conoscibile per noi e il conoscibile in senso assoluto. Perciò è necessario procedere in questo modo: da ciò che è meno chiaro per natura, ma più chiaro per noi a ciò che è più chiaro $\mathrm{e}$ conoscibile per natura (Fisica, A, 1, 184 a 15-20).

Il movimento descritto, quindi, va

1. da ciò che è meno chiaro per natura, ma più chiaro per noi, cioè è più debole ontologicamente, ma più evidente alla sensazione;

2. a ciò che è più chiaro e conoscibile per natura, cioè è stabile dal punto di vista ontologico, e quindi anche meglio conoscibile in senso assoluto, ma più lontano dalla sensazione.

Infatti, Aristotele precisa ulteriormente quest'affermazione, osservando:

Innanzitutto, a noi sono chiare ed evidenti le cose considerate nel

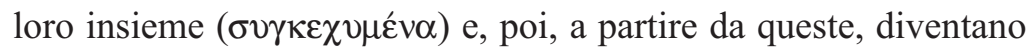
conoscibili gli elementi ( $\tau \grave{\alpha} \sigma \tau o \iota \chi \varepsilon \hat{\imath} \alpha$ ) e i principi li determinano ( $\alpha \hat{i}$

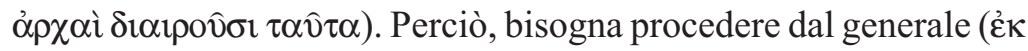

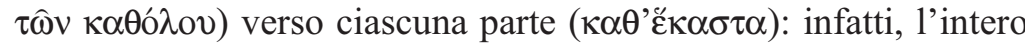

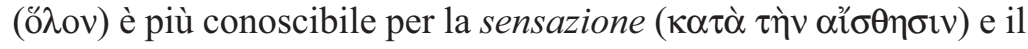




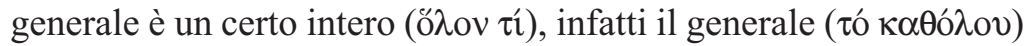
racchiude molte cose come parti. E la stessa cosa avviene, in un certo senso, anche ai nomi rispetto alla definizione: questi, infatti, indicano un certo intero e in modo indeterminato, per esempio il 'cerchio', la sua definizione lo determina in ciascuna accezione (184 a $21-184$ b 12).

Quella che potremmo definire la teorizzazione di questa distinzione tra ciò che è più chiaro per noi o per natura, la troviamo in un passo degli Analitici Secondi, A, 2, nel quale Aristotele afferma:

Ciò che è primo e più conoscibile si dice in due modi ( $\delta \chi \chi \hat{\omega} \varsigma)$ : non è infatti la stessa cosa primo per natura e primo per noi, né più conoscibile e più conoscibile per noi: dico primo e più conoscibile per noi ciò che è più vicino alla sensazione, ma primo e più conoscibile in senso assoluto ciò che è più lontano $(71 \mathrm{~b} 33-72 \mathrm{a} 4)$.

Ora, però, nel contesto degli Analitici, gli stessi termini

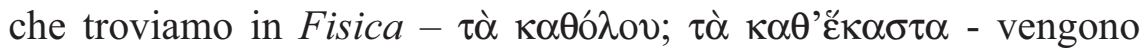
semantizzati in maniera opposta rispetto a quanto si è appena visto:

sono più lontane soprattutto le cose universali ( $\tau \grave{\alpha} \kappa \alpha \theta \dot{\lambda} \lambda o v)$, più vicine le particolari ( $\tau \grave{\alpha} \kappa \alpha \theta$ 'ย́ $\kappa \alpha \sigma \tau \alpha$ ) e queste si oppongono tra di loro: dai primi deriva ciò che dipende dai principi propri: infatti è

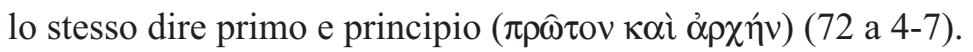

E' evidente dai passi appena citati che il contesto nel quale Aristotele pone e spiega questi termini è molto diverso: se, infatti, negli Analitici lo Stagirita sta descrivendo il sillogismo scientifico e, dunque, argomenta intorno alla natura di una scienza dimostrativa che si muove dai principi alle conclusioni, nella Fisica «sta descrivendo il metodo per ottenere la conoscenza degli $\alpha \rho \chi \alpha$ í... Questo è proprio l'opposto della dimostrazione scientifica, è il 
metodo di risalire da ciò che è confusamente dato nell'esperienza a ciò che presuppone ${ }^{60}$. Il punto di vista predominante in Fisica,

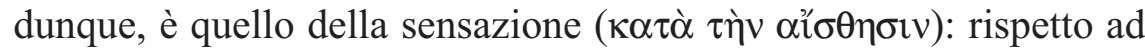
essa si misura il più chiaro e il più conoscibile. Qui si sta, infatti, descrivendo un procedimento euristico, con una forte componente empirica, procedimento che, in quanto tale, si muove a partire da ciò che si presenta alla sensazione fino alle cause e ai principi, cioè agli elementi che costituiscono il generale indistinto che appare alla sensazione. Negli Analitici Secondi si descrive, invece, un movimento opposto, proprio di un procedimento dimostrativo, che, in quanto tale, si muove dai principi alle conclusioni.

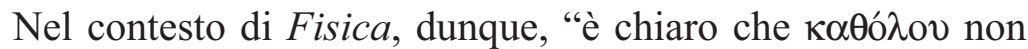
è usato nel suo normale significato. Il riferimento deve essere non a un universale concepito molto chiaramente nella sua vera natura, ma a quel grado di conoscenza nel quale si riconosce, attraverso la percezione, che un oggetto possiede una certa caratteristica generale". ${ }^{11}$ Generale, dunque, è un intero composto di parti e, su questa linea, nel $\kappa \alpha \theta$ 'ย̌ $\alpha \sigma \sigma \tau \alpha$ è possibile riconoscere le singole parti - fino a risalire agli elementi primi - che compongono l'intero dato alla nostra percezione. ${ }^{62}$

Il senso particolare in cui va intesa, in questo contesto,

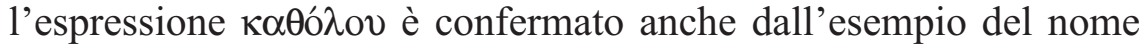
e della definizione: Aristotele sembra infatti indicare nel nome una sorta di intero che può riferirsi a più realtà e che viene, dunque,

\footnotetext{
${ }^{60}$ Ross, Aristotle's Physics..., pp. 456-457.

${ }^{61}$ Ross, Aristotle's Physics..., p. 457.

${ }^{62}$ Dato il contesto, questa lettura mi sembra più corretta di quella proposta da Ross (Aristotle's Physics..., p. 458) che afferma che l'espressione "sembra avere qui un significato non usuale: cioè sta a significare i vari sensi di un termine ambiguo". Dato che, però, Aristotele sta parlando di un к $\alpha \theta$ ó $\lambda$ ov da intendere come intero mi sembra più probabile pensare che l'espressione $\kappa \alpha \theta$ 'ย̌ $\kappa \alpha \sigma \tau \alpha$ si riferisca alle parti, cioè a ciò che ha reso possibile l'intero.
} 
precisato in modo particolare dalla definizione. ${ }^{63}$

Dunque, prima di tutto, attraverso la sensazione, si è

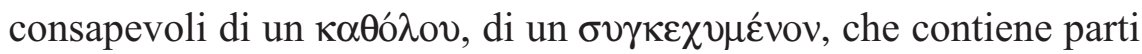
indistinte, poi si risale agli elementi costitutivi di tale intero ed è possibile distinguerne i principi.

A ulteriore dimostrazione della duttilità metodologica tipica di Aristotele, è rilevante notare che tale metodo induttivo, definito come proprio della scienza fisica, nelle linee iniziali della Fisica ( $\pi \dot{\varphi} \varphi v \kappa \varepsilon$

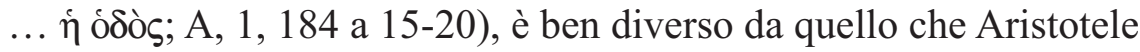
descrive in Fisica, A, 1, 184 a 10-16, come metodo che permette di avere scienza di un dato oggetto. In quel contesto, infatti, come si è visto, lo Stagirita delinea e applica un procedimento dialettico attraverso cui risalire ai principi primi di una scienza, tramite la discussione con le opinioni dei predecessori, perché si ha scienza, quando dalle cause e dai principi si scende alle cose particolari.

E' evidente che in questo modo si descrive un procedimento scientifico dimostrativo vicino a quello descritto negli Analitici, per cui è diverso anche il metodo che si applica per perseguirlo. Inoltre, questa discussione con i predecessori sui principi primi si pone come preparatoria alla scienza fisica e, quindi, neanche strettamente fisica, il che giustifica ulteriormente la variazione di metodo e non comporta contraddizione: infatti, se il metodo più propriamente fisico è quello induttivo, questo, come si è visto, non esclude affatto altri approcci metodologici, come quello dialettico.

B. Dalle proprietà del genere alle caratteristiche della specie: il De partibus animalium

Nel contesto propriamente biologico del De partibus

${ }^{63} \mathrm{Il}$ contrasto tra l'intero e il particolare "viene illustrato attraverso il contrasto tra il nome e la definizione, cioè tra l'uso del nome unito a una generale conoscenza delle caratteristiche che esso indica, e l'uso della definizione che mostra più chiaramente il significato del nome" (Ross, Aristotle's Physics..., p. 457).

Educ. e Filos. Uberlândia, v. 23, n 45, p. 101-164, jan./jun. 2009. 
animalium, troviamo un senso diverso e più connotato tecnicamente

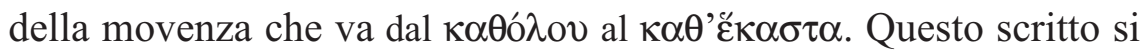
apre proprio intavolando una discussione sul metodo.

Rispetto ad ogni conoscenza e ad ogni ricerca - tanto la più umile quanto la più pregevole - si presentano due tipi di condizione ( $\left.\xi^{\prime} \xi \varepsilon \omega \varsigma\right)$, uno dei quali è correttamente considerato $<1>$ la scienza

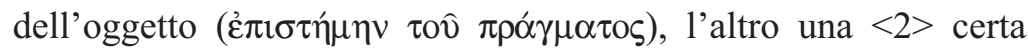

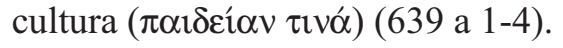

Il discrimine tra questi due tipi di conoscenza sembra individuato da Aristotele rispetto all'estensione del sapere: infatti, egli afferma che può definirsi colto l'uomo che sa distinguere, in un'esposizione, ciò che è detto in modo corretto e scorretto e di un tale uomo si dice appunto che ha una cultura generale,

ma soltanto questi consideriamo per così dire individualmente capace di giudicare riguardo a tutto, altri invece rispetto a una certa natura determinata: ci può infatti essere qualcuno che si trova nello stesso stato del primo di cui si è parlato, ma rispetto a una parte

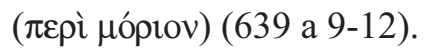

A questo punto, Aristotele sembra rendere più complesso il quadro, perché la distinzione di queste due condizioni di sapere è operata tramite due assi che si intersecano:

1. esteso o particolare;

2. specifico o generico;

Dunque, in ogni campo del sapere, si può avere $<1>$ una episteme accompagna a paideia (estesa e specifica) che permette di vagliare tutto, e $<2>$ una episteme applicata ad una singola parte (particolare e specifica). E' chiaro che se questa distinzione tra le diverse forme o estensioni del sapere riguarda ogni campo d'indagine, 
anche nella ricerca naturale devono esserci criteri con i quali poter valutare la forma delle esposizioni, "a prescindere dalla questione se la verità stia in quel modo o in un altro" (639 a 15).

Questa frase esplicita il fatto che Aristotele qui sta solo parlando di metodo, per così dire di forma della ricerca, a prescindere dal suo contenuto.

Il problema dell'impostazione della ricerca, nel campo naturale $-\mathrm{e}$ in particolare biologico - viene ritradotto in questi termini:

[...] dico, per esempio, se, considerando ogni singola sostanza, si debba definirla per se stessa (trattando uno per uno la natura dell'uomo o del leone o del bue o di qualche altro animale), oppure (se si debba definire) prendendo come fondamento gli attributi comuni ad ogni animale secondo un punto di vista comune (639 a 15-19).

Ci sono, infatti, attributi comuni ai diversi generi di animali come il sonno, la respirazione, l'accrescimento, il decadimento, la morte e ancora tutte le altre funzioni e condizioni di tal genere. Stando così le cose, se si trattassero separatamente i diversi gruppi di animali, si ripeterebbero spesso le stesse cose. Ci sono, poi, altri attributi che, pur avendo la stessa denominazione generale, differiscono per specie, come la locomozione degli animali che si differenzia rispetto alla forma: gli animali si possono muovere volando, nuotando, camminando o strisciando.

Questi sono i termini del problema e rispetto ad esso, conclude Aristotele,

[...] non deve rimanere oscuro il modo in cui bisogna ricercare, se per prima cosa si debba analizzare ciò che è comune secondo

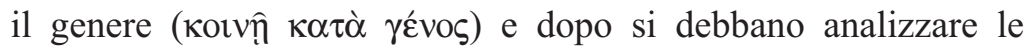
particolarità proprie, oppure se bisogna subito analizzare specie

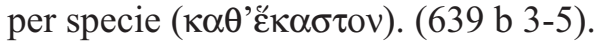


Quindi, nella trattazione biologica il discrimine è tra un sapere esteso, cioè che prenda in considerazione ciò che è comune ad ogni genere e un sapere particolare, che consideri invece separatamente le singole specie. In questo caso, Aristotele sta articolando, in senso ancora diverso, la movenza che va dal generale al particolare. Infatti, qui, diversamente da quanto si è visto finora, per generale si intendono "le caratteristiche comuni dei generi" e per particolare si intendono le "particolarità specifiche".

Dopo aver esplicitato questo problema, Aristotele pone un'ulteriore questione che è comunque legata a questa e che, come la precedente, i predecessori non hanno saputo risolvere:

[...] se, come i matematici che fanno dimostrazioni di astronomia, così anche il fisico debba analizzare per prima cosa ciò che è evidente ( $\tau \grave{\alpha} \varphi \alpha \imath v o ́ \mu \varepsilon v \alpha)$ in relazione agli animali e le parti di ognuno di essi, poi dire il perché e le cause, oppure (procedere) in qualche altro modo? (639 b 7-10).

Il problema si sposta, quindi, sul punto di partenza della ricerca e si riconoscono due possibili movenze: 1 . si può partire dalla descrizione della generazione naturale di ogni animale o 2. si può descrivere come l'animale è.

Non si deve neanche trascurare il problema se convenga esporre come ciascun (animale) si è naturalmente generato, secondo il modo in cui i predecessori conducevano la speculazione, piuttosto che dire come esso è: questo procedimento, infatti, differisce non poco da quello (640 a 10-12).

Una tale distinzione corrisponde a chiedersi se il procedimento debba partire dalle cause (in questo caso, infatti, la generazione naturale di ogni animale è la causa, come Aristotele chiarisce nel passo sottostante) o risalire a tali cause a partire dall'osservazione 
di ogni animale. Implicitamente vediamo dunque riproposta, la distinzione già vista in Fisica: dai fenomeni osservati (da ciò che è primo per la sensazione) si risale alle cause e Aristotele ribadisce che, in campo naturale, il procedimento più appropriato è proprio quello induttivo:

[...] sembra che, come è stato detto anche in precedenza, si debba cominciare da qui, cioè che per prima cosa si debba raccogliere ciò che è evidente ( $t_{\mid}^{\prime}$ fainÒmena) in relazione a ciascun genere e che poi si debba trattare delle loro cause ( $\mathrm{t} j \mathrm{j}$ a,,t...aj) e della generazione (640 a 10-15).

A/B. Nota sul valore dell'esperienza nell'approccio induttivo della Fisica e del De generatione animalium

La movenza di risalita dai fenomeni ai principi e alle cause che Aristotele, in diversi luoghi, definisce come più propria della

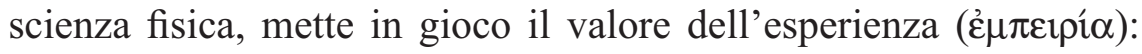
infatti, è "primo per noi" ciò che conosciamo attraverso la sensazione e, dunque, l'esperienza che si ha di un oggetto, è uno dei punti di partenza (certo non il solo, come dimostra la costante abitudine di discutere gli endoxa dei predecessori) della scienza fisica ed è forse quello che meglio la caratterizza.

Nel De generatione et corruptione, tramite un confronto stretto tra Platone e gli Atomisti (che si pone nel contesto della critica aristotelica alla dottrina platonica delle superfici), Aristotele giudica molto severamente l'approccio platonico alla fisica, evidenziando una profonda differenza, da questo punto di vista, con il suo maestro e offrendoci, in negativo, la sua opinione circa gli "strumenti" adeguati per affrontare le questioni fisiche.

Infatti, gli atomisti "hanno una maggiore dimestichezza con i fatti fisici, sono più capaci di porre quei principi che permettono di spiegare un maggior numero di realtà", mentre ai platonici si 


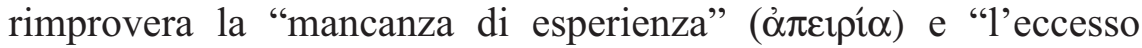
di dialettica" ( $\dot{\kappa} \kappa \hat{\omega} \nu \pi 0 \lambda \lambda \hat{\omega} v \lambda{ }^{\prime} \gamma \omega v$ ) che li porta a conclusioni raggiunte con troppa faciloneria (A, 2, 316 a 5-10).

Questa critica trova un naturale sviluppo e un'ulteriore spiegazione in un vicino contesto critico che riguarda l'esclusione, da parte di Platone, della terra dal ciclo di generazione reciproca degli elementi (De caelo, Г, 7, 306 a; cfr. Timeo, 54 B). Aristotele osserva che una tale posizione è insostenibile sia sul piano del ragionamento sia su quello dell'esperienza sensibile e, analogamente a quanto si è appena visto, rimprovera ai platonici di proporre tesi che non concordano con i fenomeni. In questa espressione si esplicita, parzialmente, la critica rivolta a Platone nel confronto con gli atomisti. Infatti, questa rilevata mancanza di rispondenza tra le teorie e i fenomeni riecheggia l'osservazione secondo la quale il filosofo ateniese tiene poco conto dell'esperienza.

In questo passaggio, inoltre, Aristotele approfondisce la sua analisi, tentando di giustificare tale sfasamento tra teoria ed esperienza; egli individua nel procedimento platonico un errore a monte: i platonici sbagliano già nel porre i loro principi primi, perché pretendono di ricondurre ad essi ogni cosa sulla base di determinate opinioni, come quella che la terra si distingua per eccellenza dagli altri elementi ( $\Gamma, 7,306$ a 7-9). Dunque essi tengono fermi i principi che stabiliscono, convinti che siano veri, accettando ogni conseguenza che da questi derivi, mentre i principi dovrebbero venir giudicati soltanto dalle conseguenze e, dunque, dal risultato finale che raggiungono. Qui, allora, si ha una chiara esplicitazione del giudizio espresso nel De generatione et corruptione: i platonici eccedono nella dialettica e mancano di esperienza, perché, appunto, sono attaccati alle loro opinioni e le difendono ad ogni costo $\left(\Gamma, 7,306\right.$ a 11-15). ${ }^{64}$ Essi, dunque, non

64 "Potremmo pensare ad Aristotele come a colui che ha unito all'analisi deduttiva e matematica della scienza platonica, la consapevolezza secondo la quale la validità di una scienza della natura viene testata, in ultima analisi, dall'osservazione empirica" (I.E. Drabkin, Notes on the laws of motion in Aristotle, "American 
sono "paradigmatici" in modo coerente, perché verificano i principi solo sul piano della coerenza interna e non su quello, fondamentale in fisica, della verifica a livello fenomenico.

Lo Stagirita suggerisce, invece, che il banco di prova della teoria e dei principi fisici sono proprio i fenomeni e l'esperienza, perché per la scienza della natura il fine ( $\tau \varepsilon ́ \lambda o \varsigma)$ è "ciò che si manifesta

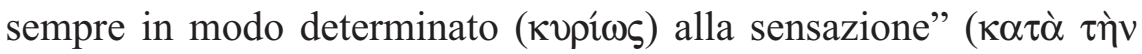
$\alpha i ̋ \theta \eta \sigma \mathrm{v})$ (306 a 16-17)..

Dunque, in questi passaggi, lo Stagirita traccia le coordinate metodologiche fondamentali entro cui il physikos deve muoversi:

1. considerazione dell'esperienza sensibile che, in un passo della Metafisica, viene descritta come un processo innescato dalla memoria attraverso ripetute percezioni sensibili:

[...] pertanto, mentre gli altri animali vivono con immagini sensibili

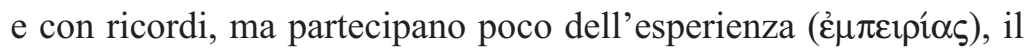
genere umano vive anche d'arte e di ragionamenti. Negli uomini l'esperienza deriva dalla memoria: infatti, molti ricordi dello stesso oggetto costituiscono la potenza di un'esperienza unica

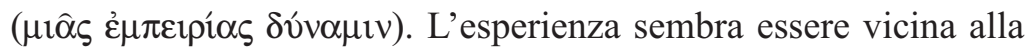
scienza e simile all'arte: in effetti, gli uomini acquistano scienza e arte attraverso l'esperienza (Metafisica, A, 1,980 b $25-981$ a 2) ${ }^{65}$.

\footnotetext{
Journal of Philology" 59 (1938), pp. 60-84, p. 82). Bisogna notare, però, che, in alcuni casi, la stessa critica che Aristotele rivolge a Platone potrebbe rivolgerla a se stesso, perché, nelle sue ricostruzioni, talvolta anch'egli cede ad esigenze di ordine sistematico che conducono a risultati paradossali rispetto al principio metodologico dell'esperienza. A questo proposito, cfr. Migliori, Ontologia..., p. 101, il quale porta l'esempio della trattazione degli elementi, in cui "la caratteristica di fondo dell'acqua diviene il freddo e non l'umido attribuito invece all'aria".

${ }^{65}$ Mansion (Introduction..., p. 158) osserva, a questo proposito, che "la fisica (aristotelica) trae i suoi principi primi attraverso la mediazione di dati fenomenici forniti dalla percezione dei sensi".
} 
2. Capacità di porre un principio che spieghi - e dunque sia in accordo con - più fenomeni.

Ad ulteriore dimostrazione dell'importanza del dato d'esperienza nella scienza fisica consideriamo la duplice argomentazione che Aristotele svolge nel De generatione animalium (nel quadro di una discussione riguardante il caso particolare dei muli che, a differenza di tutte le altre specie animali, presentano una sterilità estesa a tutta la specie).

Innanzitutto, lo Stagirita dà una spiegazione razionale del fenomeno, affermando "la chiamo razionale ( più è universale, tanto più si allontana dai principi particolari” $(\mathrm{B}, 8$, 747 b 28-30).

In questo senso, dunque, essa sembra coincidere con il punto di vista generale che Aristotele indica con il termine $\kappa \alpha \theta$ ó $\lambda$ ov. Infatti, subito di seguito, egli osserva che questo è un discorso troppo universale che, in quanto tale, rischia di diventare insensato e conclude che "in questo modo, perciò, non bisogna svolgere né alcun'altra indagine, né quella che riguarda la natura" $(\mathrm{B}, 8,748 \mathrm{a}, 13-14)$.

In generale, dunque, una ricerca che si basi soltanto su concetti universali potrebbe staccarsi troppo dalla realtà che deve giustificare ed è per questo che, soprattutto nell'ambito della scienza della natura, è legittimo affiancare, a questo punto di vista, un ulteriore e diverso punto di vista che sia più attinente all'oggetto indagato. Dunque, sulla base di questa considerazione, lo Stagirita passa ad osservazioni su ciò che è proprio del genere dei cavalli e degli asini, cioè ad un'argomentazione che si può a buon diritto chiamare physikōs, perché, appunto, è legata alla particolare natura di questi animali e non più ad argomenti di respiro universale.

Dunque, si ripropone qui lo stesso schema bipartito tra physikōs e katholou che abbiamo visto operante con costanza nel 
De caelo $;{ }^{66}$ in questo passaggio, inoltre, lo Stagirita sembra fornire una ragione metodologica per l'applicazione di questi due punti di vista (e, in particolare, ovviamente per il physikōs) che risulta in linea con le osservazioni critiche rivolte a Platone. Infatti, egli qui suggerisce che, per quanto riguarda la scienza della natura, non basta giustificare i fenomeni dal punto di vista razionale o logico, ma essi devono rispondere a criteri fisici, cioè, appunto, devono accordarsi con i fenomeni.

E' evidente, comunque, da questo esempio come questi due punti di vista - e i diversi approcci metodologici che sottintendono - non siano affatto posti come alternativi: sono due strade entrambe possibili e che, anzi, lo Stagirita pone in un rapporto di collaborazione. il De anima

C. Dall'anima in generale alle funzioni particolari dell'anima:

Un'ulteriore articolazione della movenza "dal generale al particolare" si trova nel De anima. In questo contesto, il valore di tale approccio metodologico risulta in parte confrontabile sia a quanto si è visto in Fisica, sia alla distinzione operata nel De partibus animalium tra ciò che è comune e ciò che è particolare, anche se si perde il senso tecnico visto sopra. Qui, infatti, Aristotele opera una costante "calibratura dell'argomentazione", passando da una considerazione generale di anima ad una definizione particolare delle parti o funzioni dell'anima.

Il concetto di generale conserva, dunque, il carattere dell'indistinzione, ma, nel caso della definizione generale dell'anima, non è ovviamente messa in gioco la sensazione; analogamente, le funzioni particolari dell'anima non rappresentano i principi, ma caratteristiche o funzioni costitutive che vanno distinte, raffinando via via la definizione generale di anima.

\footnotetext{
${ }^{66}$ cfr., in particolare, De caelo, A, 10, 280 a 32-34 e paragrafo 4, pp. ***. 
In De anima, B, 1 Aristotele propone, infatti, una ricerca che porti a definire l'anima in generale:

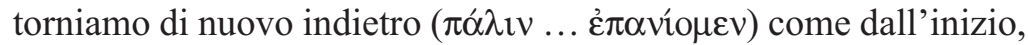
tentando di determinare che cos'è l'anima e qual è la sua definizione

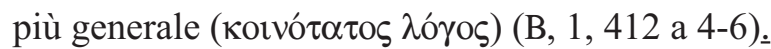

Tale ricerca porta a concludere che l'anima è 'l'atto primo di un corpo naturale che funziona come strumento (ỏpyovıkóv)" ${ }^{\prime 67}$ (B, 1, 412 b), infatti lo Stagirita chiude il libro primo affermando "Come abbozzo ( $\tau \dot{\pi} \pi \omega)$ l'anima sia definita e descritta cosi”" (B, 1, 413 a 9-10).

Lo scenario cambia, al paragrafo 2 del secondo libro, in cui si annuncia un nuovo tipo di ricerca e, dunque, un nuovo inizio: ${ }^{68}$

Poiché da ciò che è più indistinto (

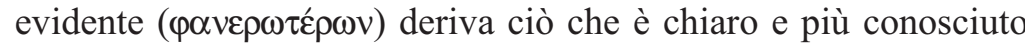

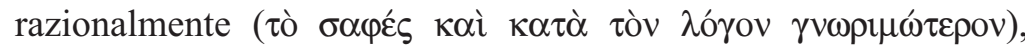
bisogna di nuovo cercare di considerare così (ơั $\tau \omega)$ l'anima. Infatti, bisogna che il discorso che definisce non chiarisca soltanto

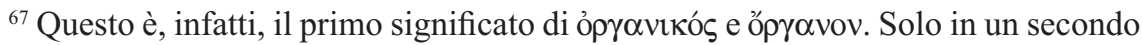

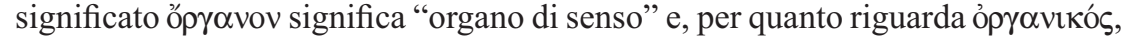
il senso di "dotato di organi" non è attestato (Cfr. P. Chantraine, Dictionnaire etymologique de la langue grecque. Histoire des mots, Klincksieck, Paris, 1984, p. 815; H.G. Liddell - R. Scott, Greek English Lexicon, Clarendon Press, Oxford, 1968, p. 1245). Concordo, dunque, con la lettura che di questa definizione dà Bos (A.P. Bos, The Soul and its instrumental Body. A reinterpretation of Aristotle's Philosophy of Living Nature, Brill, Leiden - Boston, 2003, p. 6) il quale afferma che, se si intende la definizione aristotelica, nel senso di un "corpo dotato di organi", Aristotele sarebbe in rottura con le teorie psicologiche a lui legate, compresa quella di Platone e anche con se stesso. Inoltre, tradurre con "dotato di

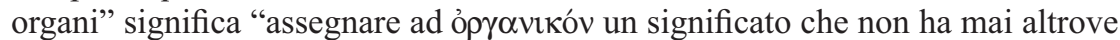
in Aristotele" (A.P. Bos, Why the soul needs instrumental body according to Aristotle, «Hermes» 128 (2000), pp. 20-31, p. 25).
}

68 Il fatto che si è avviata una nuova ricerca viene ribadito anche in seguito: "diciamo, pertanto, riprendendo la ricerca dall'inizio [...]" (B, 2, 413 a 20-21). 
$<1>$ il che (ö $\tau$ ), come la maggior parte delle definizioni, ma deve comprendere e rendere evidente $<2>$ anche la causa ( $\alpha i \tau i \alpha v)$ (B, 2, 413 a 11-16).

La distinzione qui operata tra ciò che è incerto e, allo stesso tempo più evidente, e ciò che è più conosciuto secondo ragione, ricorda la distinzione, teorizzata in termini simili, nel primo libro della Fisica tra ciò che è più chiaro in sé e per noi, ma in questo contesto tale distinzione è funzionale a descrivere un processo analitico, più che induttivo, che rimanda alle parti o funzioni dell'anima.

In questo passaggio, Aristotele distingue due processi di conoscenza:

1. un processo definitorio di semplice descrizione;

2. un processo che sia capace di risalire anche alle cause;

Ora, il discorso intorno ad un oggetto deve superare il processo di semplice definizione (1), per poter indicare le cause (2), appunto perché si deve andare da ciò che è più evidente a ciò che è più conosciuto razionalmente. Proprio questa necessità di risalire verso le cause configura, infatti, la ricerca come un andare da ciò che

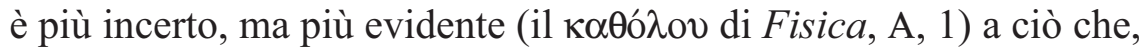
invece, è più chiaro razionalmente (il $\kappa \alpha \theta$ 'ह̌ $\alpha \alpha \sigma \tau \alpha$ di Fisica).

Questa nuova ricerca porta Aristotele a considerare le anime particolari o le diverse funzioni dell'anima. Queste definizioni sono ritenute più adeguate rispetto a quella generale, nel caso dell' anima come in quello della figura:

E' chiaro pertanto che, allo stesso modo, una è la definizione di anima e di figura. Infatti, in quest'ultimo caso, non esiste una figura oltre il triangolo e le figure che seguono, nel primo caso, non esiste un'anima oltre quelle dette. Potrebbe esserci una definizione comune per le figure che si adatterà a tutte, ma non sarà propria di nessuna figura; lo stesso discorso vale per le anime di cui si è parlato. Perciò è ridicolo, sia in questi sia in altri casi, cercare la 
definizione comune che non sarà la definizione propria di nessuna realtà (B, 3, 414 b 20-27).

\section{Conclusioni}

L'esemplificazione qui proposta ci ha messo di fronte sia a una pluralità di "oggetti propri" della fisica e di punti di vista dai quali si può conoscere una stessa realtà sia, di conseguenza, a una pluralità di approcci metodologici alle questioni fisiche.

In particolare, l'esame di alcuni passi delle diverse opere fisiche e biologiche ci ha permesso di riscontrare la compresenza di due approcci molto diversi - che assumono punti di partenza diversi e hanno finalità diverse -, a loro volta articolati in più modi o applicati a contesti diversi:

1. il metodo dialettico, basato sull'analisi degli endoxa dei predecessori: permette di rilevare le aporie e di superarle; inoltre-se applicato non a semplici tesi, ma alla discussione intorno ai principi - può anche essere funzionale ad una definizione dei principi primi di una data scienza;

2. un approccio che potremmo definire induttivo che procede cioè dal generale, inteso come intero indistinto o come ciò che è comune, ai particolari, intesi come (a) elementi di cui l'intero è costituito o (b) come principi o come (c) parti e funzioni. E' un processo che, generalmente, parte dalla sensazione o comunque da ciò "che è primo per noi" (quindi, non dagli endoxa) e risale ai principi, nel contesto di una scienza euristica che, a partire dall'esperienza di ciò che risulta evidente, va alla scoperta di ciò che è più chiaro e conoscibile, in senso assolto, ma non per noi.

L'approccio dialettico sembra essere soprattutto funzionale ad un procedimento scientifico dimostrativo che dai principi 
discende a giustificare le realtà particolari. L'analisi degli endoxa permette, infatti, di stabilire quale sia la teoria che meglio sa spiegare un dato fenomeno (che quindi deve essere conosciuto). In questo senso, questo approccio metodologico svolge una funzione critico-costruttiva nei confronti dei predecessori e permette, inoltre, di individuare e discutere alcuni problemi sottesi alle loro soluzioni. L'approccio che abbiamo definito induttivo, invece, sembra più proprio di una scienza concepita nella sua funzione euristica, che, dunque, dall'intero o da ciò che è comune - e che, in forza di questo, si presenta come primo alla sensazione ed evidente - risale agli elementi che costituiscono e causano tale intero.

Il secondo è il metodo che in Fisica è definito come propriamente fisico, ma, come si è visto, in ogni opera fisica (fatta eccezione per i Parva Naturalia e per alcune opere biologiche molto tecniche) il metodo dialettico è operante e trova una sezione ad esso dedicata.

Aristotele, dunque, non sembra affatto porre come alternativi (e autoescludentesi) ${ }^{69}$ questi due metodi, cosa evidente anche dal fatto

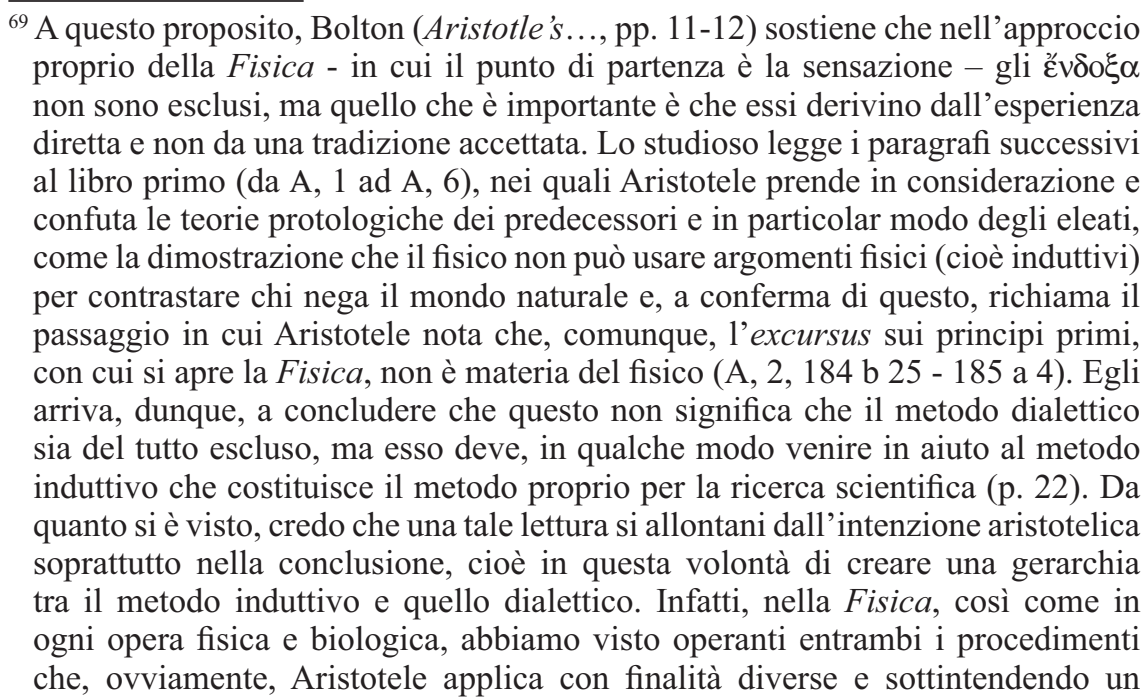

Educ. e Filos. Uberlândia, v. 23, n 45, p. 101-164, jan./jun. 2009. 
che, come si avuto modo di verificare, nei trattati fisici e biologici, molto spesso contrappone "i ragionamenti" ai "fatti" e con costanza osserva lo stesso fenomeno dai due punti di vista, anche se la cifra distintiva della scienza fisica si trova nell'accordo tra fenomeni e principi: quindi, la dimensione empirica ha sicuramente un peso maggiore che in altre scienze.

In questo senso, dunque, è evidente, che non esiste, $i l$ metodo aristotelico per le opere fisiche, come noi,

[...] figli di Cartesio e certi dell'unità del metodo scientifico" potremmo intenderlo, perché "questo non si dà nel pensiero antico che invece privilegia la continua indagine sui molteplici irriducibili aspetti che la realtà multiforme ci propone ed è disponibile ad una pluralità di ipotesi e di tentativi, cioè di 'vie', che, di volta in volta, possono essere, con risultati diversi, legittimamente percorse $[\ldots]$ È importante capire il senso di questo pluralismo 'metodico'. Se si costruisce la filosofia non per una volontà di avere idee chiare e distinte organizzate in un sistema che ha come fondamentale e necessaria caratteristica l'assoluta coerenza, ma per il bisogno di

procedimento scientifico diverso nei due casi. Dunque, il metodo dialettico e il metodo induttivo sembrano sì essere posti in un rapporto di collaborazione (anche se non sempre né necessariamente), ma allo stesso titolo, appunto perché ad essi si riconoscono funzionalità e contesti di applicazione diversi. Anche nell'interpretazione che dà Zanatta (M. Zanatta, Aristotele, Fisica, U.T.E.T., Torino, 1999, p. 42) dell'incipit della Fisica sembra pesare la necessità di imporre una scelta netta tra il metodo dialettico e il metodo induttivo: egli, infatti, vede nel metodo che da ciò che è primo per noi procede fino a ciò che è primo per natura "un'altra importante strategia del metodo dialettico posta in atto dalla fisica" che consiste nell'analisi linguistica. Secondo questa lettura, nel descrivere il procedimento di successiva divisione dal generale al particolare, Aristotele si riferirebbe ad un'operazione di analisi linguistica e l'esempio del nome e della definizione lo confermerebbe. Il tentativo sembra dunque quello di ricondurre il metodo induttivo a quello dialettico. Come abbiamo visto, però, in ambito fisico, il cosiddetto metodo induttivo opera tanto quanto quello dialettico e anzi, è detto il più proprio della scienza fisica. E' evidente, dunque, che la scelta di un metodo univoco non si pone affatto per Aristotele.

Educ. e Filos. Uberlândia, v. 23, n 45, p. 101-164, jan./jun. 2009. 
capire una realtà di cui si riconosce la poliedricità, diviene utile, se non necessario, costruire schemi diversi, scontando anche un loro problematico rapporto reciproco. Se si assume questo punto di vista l'unità del metodo è un absurdum o almeno un ostacolo all'indagine. $^{70}$

Un'affermazione questa, che Aristotele condividerebbe nella sua critica all'eccesso di dialettica che rimprovera ai platonici.

In quest'ottica trova piena ragione e giustificazione il quadro frastagliato che risulta dalla nostra analisi e che, infatti, ci costringe a parlare di diversi metodi aristotelici - presenti e operanti a pieno titolo in ogni opera fisica - che sembrano adattarsi di volta in volta non solo alla realtà che è oggetto di analisi, ma anche ai diversi contesti e alla diversa prospettiva e finalità dell'analisi stessa.

\section{Bibliografia}

Aristotele, Meteorologia, Introduzione, traduzione, note e apparati di L. Pepe, Bompiani, Milano, 2003.

Aristotele, La generazione e la corruzione, traduzione, introduzione e commento di M. Migliori, Loffredo Editore, Napoli, 1976.

Aristotele, Metafisica, a cura di G. Reale, Rusconi, Milano, 1993.

Aristotele, L'anima, a cura di G. Movia, Loffredo Editore, Napoli, 1979.

Aristotele, Opere biologiche, a cura di D. Lanza e M. Vegetti, U.T.E.T., Torino, 1971.

Aristotle's Physics, a revised text with introduction and commentary by W.D. Ross, Clarendon Press, Oxford, 1936, $1955^{2}$.

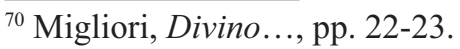

Educ. e Filos. Uberlândia, v. 23, n 45, p. 101-164, jan./jun. 2009.

160
} 
Aristotele, Fisica, a cura di M. Zanatta, U.T.E.T., Torino, 1999.

Ackrill J.L., Aristotle the Philosopher, Oxford University Press, Oxford, New York, Toronto, 1981; traduzione italiana di P. Crivelli, Aristotele, Il Mulino, Bologna, 1993.

Berti E., Nuovi studi aristotelici. Epistemologia, logica e dialettica, volume I, Morcelliana, Brescia, 2004.

Berti E., Nuovi studi aristotelici. Fisica, antropologia e metafisica, volume II, Morcelliana, Brescia, 2005.

Bolton R., Aristotle's Method in Natural Science, in Aristotle's Physics: a collection of Essays, Edited by L. Judson, Clarendon Press, Oxford, 1991.

Bos A.P., The Soul and its instrumental Body. A reinterpretation of Aristotle's Philosophy of Living Nature,

Brill, Leiden - Boston, 2003.

Bos A.P., Why the soul needs instrumental body according to Aristotle, «Hermes» 128 (2000), pp. 20-31.

Brunschwig J., Qu'est ce que "la physique” d'Aristote?, in AA. VV., La Physique d'Aristote e les conditions d'une science de la nature, Actes du Colloque organisé par le Séminaire d'Epistémologie et d'Histoire des Sciences de Nice, Edités par F. De Gandt et P. Souffrin, Librairie Philosophique J. Vrin, Paris, 1991, pp. 11-37.

Callahan J.F., Four views of time in ancient philosophy, Harvard University Press, Cambridge, 1948.

Cattanei E., Enti matematici e metafisica. Platone, l'Accademia e Aristotele a confronto, Vita e pensiero, Milano, 1996. 
Chantraine P., Dictionnaire etymologique de la langue grecque. Histoire des mots, Klincksieck, Paris, 1984.

Donini P., La filosofia di Aristotele, Loescher, Torino, 1982.

Drabkin I.E., Notes on the laws of motion in Aristotle, «American Journal of Philology» 59 (1938), pp. 60-84.

Hankinson R. J., Science, in AA. VV., The Cambridge Companion to Aristotle, edited by J. Barnes, Cambridge University Press, 1995.

Hicks R. D., Aristotle, De anima, with translation, introduction and notes by R.D. Hicks, Cambridge University Press, Cambridge, 1907; ristampe Amsterdam, 1965; Hildesheim, 1990.

Kahn C., La Physique d'Aristote et la tradition greque de la philosophie naturelle, in AA. VV., La Physique d'Aristote e les conditions d'une science de la nature, Actes du Colloque organisé par le Séminaire d'Epistémologie et d'Histoire des Sciences de Nice, Edités par F. De Gandt et P. Souffrin, Librairie Philosophique J. Vrin, Paris, 1991, pp. 41-52.

Kahn C.H., Sensation and Consciousness in Aristotle's Psychology, in AA. VV., Articles on Aristotle, edited by J. Barnes, M. Schofield, E. Sorabji, Duckworth, London, 1979.

Liddell H.G.- Scott R., Greek English Lexicon, Clarendon Press, Oxford, 1968.

G.E.R. Lloyd, Aristotle. The Growth and Structure of His Thought, Cambridge University Press, 1968; traduzione italiana di M. Fantuzzi, Aristotele. Sviluppo e struttura del suo pensiero, Il Mulino, Bologna, 1985. 
Mansion A., Introduction à la physique aristotélicienne, Éditions de l'Institut Supérieur de Philosophie, Louvain - La - Neuve, 1913, $1945^{2}$.

Migliori M., Rapporti tra la Metafisica e il De generatione et corruptione di Aristotele, in AA.VV., Aristotele. Perché la metafisica. Studi su alcuni concetti-chiave della "filosofia prima" aristotelica e sulla storia dei loro influssi, a cura di A. Bausola e G. Reale, Milano, 1994, pp. 377-396.

Migliori M., Ontologia e materia. Un confronto tra il Timeo di Platone e il De generatione et corruptione di Aristotele, in AA. VV., Gigantomachia. Convergenze e divergenze tra Platone e Aristotele, Editrice Morcelliana, Brescia, 2002, pp. 35-104.

Migliori, Divino e umano. L'anima in Aristotele e in Platone, in AA. VV., Mente, anima e corpo nel mondo antico, a cura di U. la Palombara e G. A. Lucchetta, Opera Editrice, Pescara, 2006, pp. 21-56.

Mignucci M., In margine al concetto di forma nella Metafisica di Aristotele, in AA.VV., Aristotele..., pp. 145-170.

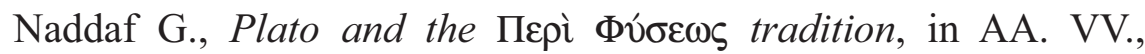
Interpreting the Timaeus - Critias, Proceedings of the IV Symposium Platonicum, Edited by T. Calvo, L. Brisson, Academia Verlag, Sankt Augustin, 1997, pp. 27-36.

Natoli S., Aristotele e la scientificità della filosofia. In genere e

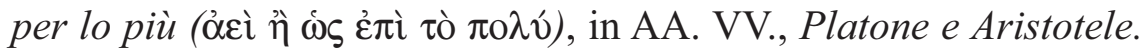
Dialettica e logica, a cura di M. Migliori - A. Fermani, Morcelliana, Brescia, 2008, pp. 295-321.

Reale G., Introduzione a Aristotele, Editori Laterza, Roma-Bari, 1974, $1991^{2}$.

Rorty A.O., De anima: its agenda and its recent interpreters, in 
M.C. Nussbaum - .O. Rorty (eds), Essays on Aristotle's De anima, Clarendon Press, Oxford, 1992.

Solmsen F., Aristotle's System of the Physical World, Cornell University Press, Ithaca, New York, 1960.

Wieland W., Die aristotelische Physik. Untersuchungen über die Grundlegung der Naturwissenschaft und die sprachlichen Bedingungen der Prinzipienforschung bei Aristoteles, Vandenhoeck \& Ruprecht, 1970; traduzione italiana di C. Gentili, La Fisica di Aristotele. Studi sulla fondazione della scienza della natura e sui fondamenti linguistici della ricerca dei principi in Aristotele, Laterza, Bologna, 1993

Data de Registro 07/05/08

Data de Aceite 10/07/08 